\title{
La diffraction des neutrons et des rayons $X$ pour l'étude structurale des liquides et des verres
}

\author{
H.E. Fischer, P.S. Salmon ${ }^{1}$ et A.C. Barnes ${ }^{2}$ \\ LURE, bâtiment 209d, Centre Universitaire Paris-Sud, BP. 34, 91898 Orsay cedex, France \\ ${ }^{1}$ Department of Physics, University of Bath, Bath BA2 7AY, U.K. \\ ${ }^{2}$ H.H. Wills Physics Laboratory, University of Bristol, Bristol BS8 1TL, U.K.
}

\begin{abstract}
Résumé: La compréhension de mainte propriété physique d'un verre ou d'un liquide nécessite la connaissance des facteurs de structure partiels (PSFs) qui décrivent chacun la distribution d'une espèce atomique autour d'une autre. La technique de diffraction des neutrons avec substitution isotopique (NDIS) $[1,2,3]$, ayant bien réussi a déterminer les PSFs de certains composés $[4,5]$, est pourtant restreinte aux isotopes présentant un contraste suffisant en longueur de diffusion. D'un autre cote, la technique de diffusion anomale des rayons X (AXS ou AXD) [6] permet de faire varier la longueur de diffusion d'une espèce atomique pourvu que son énergie d'absorption soit à la fois accessible et suffisamment élevée pour donner un assez grand transfert du moment. La combinaison des techniques de diffraction des neutrons (avec ou sans substitution isotopique) et de diffraction des rayons $\mathrm{X}$ (avec ou sans diffusion anomale) peut donc permettre d'obtenir un meilleur contraste en longueurs de diffusion pour un système donné, mais exige une analyse de données plus soignée pour pouvoir bien tenir compte des erreurs systématiques qui sont différentes pour les 2 techniques [7]. Pour les atomes ayant des distributions électroniques quasi-sphériques, e.g. dans le cas d'un alliage liquide, la combinaison des techniques de NDIS et de diffraction des rayons X s'est déjà montrée très avantageuse pour la détermination des PSFs $[8,9]$. Dans le cas des verres ayant d'importantes liaisons covalentes, l'effective combinaison des 2 techniques peut être moins directe mais facilitée lorsqu'il s'agit des atomes de grand $\mathrm{Z}[10,11]$. Nous présentons ici un sommaire du méthode et quelques exemples des résultats.
\end{abstract}

\section{SECTIONS EFFICACES ET FACTEURS DE STRUCTURE}

\subsection{Introduction}

On commence par l'équation "maitre" donnant la section efficace différentielle $d \sigma / d \Omega$ pour la diffraction d'un faisceau de quanta (e.g. rayons $\mathrm{X}$ ou neutrons) par un échantillon de $N$ atomes localisés aux sites $i$ et ayant des longueurs de diffusion $b_{i}$ (en général complexe):

$$
\frac{d \sigma}{d \Omega}=\left\langle\left|\sum_{i}^{N} b_{i} e^{i \mathbf{q} \cdot \mathbf{r}_{i}}\right|^{2}\right\rangle=\left\langle\sum_{i, j}^{N} b_{i} b_{j}^{*} e^{i \mathbf{q} \cdot \mathbf{r}_{i j}}\right\rangle,
$$

où le vecteur $\mathbf{r}_{i j}=\mathbf{r}_{i}-\mathbf{r}_{j}$ représente la position relative des sites atomiques $i$ et $j$, et le vecteur $\mathbf{q}=\mathbf{q}_{\text {sortie }}-\mathbf{q}_{\text {incident }}$ est le transfert de moment. Cette équation a l'aspect un peu complexe, mais elle dit simplement que l'atome diffuseur au site $i$ émet des ondes sphériques d'amplitude $b_{i}$ qui s'interfèrent avec celles provenant des autres sites. L'équation (1) est d'ailleurs valable pour toute morphologie de l'échantillon, que ce soit monocristalline, poudre, poreuse, liquide, verre, multicouche, matière molle, etc.

Les brackets \langle\rangle indiquent une moyenne d'ensemble sur les sites $i$ par la distribution de longueurs de diffusion présentée par chaque espèce atomique, ce qui n'est pas pertinent pour les rayons $\mathrm{X}$ car la longueur de diffusion est directement liée au nombre 
atomique $Z$. Pour les neutrons, cette distribution a deux origines possibles: une pluralité d'états de spin pour un système neutron-noyau donné, et une variation isotopique pour une espèce atomique dans l'échantillon. On verra plus tard qu'une distribution de longueurs de diffusion donne lieu à la diffusion incohérente.

Il y a une question pertinente qui mérite une petite digression: Pourquoi prendre la moyenne d'un ensemble d'échantillons lorsqu'il n'y a qu'un échantillon dans le faisceall et sa distribution de longueurs de diffusion est connaissable? Ce qui se passe en réalité est le suivant: chaque neutron ou rayon $\mathrm{X}$ a un volume de cohérence à lui, grosso modo la "taille" de son paquet d'onde qui augmente lorsque la monochromaticité ou la collimation du faisceau s'améliore. Au moment de sa diffusion quelque part dans l'échantillon, le neutron ou rayon X "voit" seulement les atomes dans son volume de cohérence. Strictement, $N$ dans l'Eq. (1) devrait donc représenter le nombre d'atomes dans un seul volume de cohérence où les amplitudes des ondes sphériques sont ajoutées en phase, et puis les $d \sigma / d \Omega$ de toutes les volumes de cohérences dans le volume de l'échantillon illuminée par le faisceau devraient être moyennés. Pour un échantillon homogène, chaque volume de cohérence est un mini-échantillon qui présente la même structure atomique que celle de ses confrères, mais elle a sa propre attribution des longueurs de diffusion sur ses atomes. Alors, si le nombre de volumes de cohérence est très important, chacun cependant assez grande pour pouvoir comprendre un bon échantillonage d'atomes, on peut remplacer une somme de l'ensemble de volumes de cohérence par la moyenne d'un ensemble d'échantillons, cette demière étant mathématiquement plus commode.

S'il n'y a qu'un volume de cohérence dans l'échantillon, c'est à dire dans le cas de la diffraction cohérente dite "speckle", on enlève les brackets de moyenne d'ensemble \langle\rangle et il faudrait tenir compte de la position exacte de chaque atome dans le seul volume de cohérence pour pouvoir calculer $d \sigma / d \Omega$.

Dans le cas de la diffraction des neutrons, la partie imaginaire d'une longueur de diffusion est généralement très petite car les résonances d'absorption sont rares (exceptions pour ${ }^{113} \mathrm{Cd},{ }^{149} \mathrm{Sm},{ }^{151} \mathrm{Eu}$ et ${ }^{157} \mathrm{Gd}$ ) et il n'est donc que rarement nécessaire de conjuguer $b_{j}$ dans l'Eq. (1). On signale aussi que cette équation "maitre" emploie l'approximation statique où l'énergie incidente du neutron $E_{\text {inc }}$ est très grande devant le transfert d'énergie $\hbar \omega$ à l'échantillon, la diffraction donnant ainsi une image quasiinstantanée de la structure (dans un volume de cohérence). Cette condition $E_{\text {inc }} \gg \hbar \omega$ est réalisé sans effort pour les rayons $\mathrm{X}$.

Par simplicité, dans ce qui suit on traitera le cas de diffraction d'un faisceau monochromatique d'une longueur d'onde $\lambda$ et non le cas d'une source pulsée de neutrons décrit ailleurs $[12,13]$. On voit que selon l'Eq. (1) la section efficace différentielle $d \sigma / d \Omega$ est fonction uniquement de q. L'intensité $I(\mathbf{q})$, i.e. les coups par seconde mesurée par une (petite) cellule de détection d'un angle solide $d \Omega$ captant les quanta ayant $\mathbf{q}_{\text {sortie }}$, est donc aussi fonction uniquement de $q$ et est donnée par:

$$
I(\mathbf{q})=\Phi \frac{d \sigma}{d \Omega} d \Omega
$$

où $\Phi$ est le flux du faisceau (d'habitude on emploie les unités suivantes: $[I]=\mathrm{s}^{-1}$, $[\Phi]=\mathrm{s}^{-1} \mathrm{~cm}^{-2},[d \Omega]=\operatorname{str}$ et $[d \sigma / d \Omega]=$ barns $/$ str où 1 barn $\left.=10^{-24} \mathrm{~cm}^{2}\right)$. Un changement de la longueur d'onde $\lambda$ du faisceau ne peut donc pas changer $I(\mathbf{q})$ mais seulement l'angle de diffraction où se trouve $\mathbf{q}_{\text {sortie. }}$. Pour un échantillon isotrope, la 
diffraction est conique (Debye-Scherrer) et l'on peut écrire:

$$
q=|\mathbf{q}|=\frac{4 \pi}{\lambda} \sin \theta
$$

où $2 \theta$ est l'angle de diffraction. En prenant l'exemple de la diffraction par un poudre, si la mesure de diffraction n'est pas "resolution-limited" et à part les effets d'attenuation par l'absorption, un changement de $\lambda$ ne change donc pas $I(q)$ mais seulement son étalement en angle $2 \theta$; c'est à dire, la largeur intrinsèque en $2 \theta$ d'un pic augmente avec $\lambda$ et donc son intensité intégrée augmente mais non son intensité $I(q)$.

Tandis que le mot "diffusion" (scattering) décrit un processus soit élastique soit inélastique, le mot "diffraction" est réservé pour le cas élastique où il n'y a pas d'analyse en échange d'énergie $\hbar \omega$ entre l'échantillon et le neutron/rayon X. Le formalisme développé dans les deux sous-sections suivantes, aussi valable pour les neutrons que pour les rayons $\mathrm{X}$, traite d'abord le cas de diffraction par un échantillon monoatomique (i.e. d'une seule espèce atomique $Z$ ) pour lequel le facteur de structure est facilement défini et directement lié à $d \sigma / d \Omega$, puis le cas d'un échantillon polyatomique. C'est seulement pour ce dernier qu'il faut considérer les facteurs de structure partiels.

\subsection{Le cas d'une seule espèce atomique (i.e. monoatomique)}

Bien qu'un échantillon monoatomique ne présente qu'un seul nombre atomique $Z$, il peut y avoir une distribution de longueurs de diffusion pour les neutrons, comme discuté ci-dessus. En supposant qu'il n'y a pas de corrélation entre les longueurs de diffusion et les positions des atomes dans l'échantillon, on peut séparer en deux facteurs le moyenne d'ensemble de l'Eq. (1), puis considérer les 2 cas:

$$
\begin{array}{ll}
\left\langle b_{i} b_{j}^{*}\right\rangle=\left\langle b_{i} b_{i}^{*}\right\rangle=\overline{b^{2}} \quad i=j \text { (meme site), } \\
\left\langle b_{i} b_{j}^{*}\right\rangle=\left\langle b_{i}\right\rangle\left\langle b_{j}^{*}\right\rangle=\bar{b}^{2} \quad i \neq j \quad \text { (sites differents), }
\end{array}
$$

et donc la section efficace différentielle devient

$$
\begin{aligned}
\frac{d \sigma}{d \Omega} & =\bar{b}^{2}\left\langle\sum_{i, j \neq i}^{N} e^{i \mathbf{q} \cdot \mathbf{r}_{i j}}\right\rangle+\sum_{i}^{N} \overline{b^{2}} \\
& =\bar{b}^{2}\left\langle\sum_{i, j}^{N} e^{i \mathbf{q} \cdot \mathbf{r}_{i j}}\right\rangle+\sum_{i}^{N}\left(\overline{b^{2}}-\bar{b}^{2}\right) .
\end{aligned}
$$

En définissant le facteur de structure (statique) $S(\mathbf{q})$ comme:

$$
S(\mathbf{q})=\frac{1}{N}\left\langle\sum_{i, j}^{N} e^{i \mathbf{q} \cdot \mathbf{r}_{i j}}\right\rangle
$$

qui est donc sans dimension, on peut ensuite écrire la section efficace différentielle par atome ainsi:

$$
\begin{aligned}
\frac{1}{N}\left[\frac{d \sigma}{d \Omega}(\mathbf{q})\right] & =\frac{1}{N}\left[\frac{d \sigma}{d \Omega}(\mathbf{q})\right]^{\mathrm{coh}}+\frac{1}{N}\left[\frac{d \sigma}{d \Omega}(\mathbf{q})\right]^{\text {incoh }} \\
& =\bar{b}^{2} S(\mathbf{q})+\left(\overline{b^{2}}-\bar{b}^{2}\right) \\
& \equiv b_{\text {coh }}^{2} S(\mathbf{q})+b_{\text {incoh }}^{2}
\end{aligned}
$$


où on montre explicitement la dépendence sur $\mathbf{q}$ uniquement. On voit donc que $d \sigma / d \Omega$ est composée d'une partie cohérente (i.e. structurée en $\mathbf{q}$ et relative à la diffraction par des points corrélés dans l'espace) et d'une partie incohérente (i.e. isotrope et relative à la diffraction par des points non-corrélés). Il est logique que $S(\mathbf{q})$, qui porte toute l'information sur la structure de l'échantillon, ne figure pas dans la contribution incohérente. La longueur de diffusion cohérente $b_{\text {coh }} \equiv \bar{b}$ est simplement la valeur moyenne de la distribution des $b$ dans l'échantillon, et la diffusion incohérente $b_{\text {incoh }}^{2} \equiv\left(\overline{b^{2}}-\bar{b}^{2}\right)=\left\langle|b-\bar{b}|^{2}\right\rangle$ est simplement la variance de cette distribution (évidemment nulle dans le cas de diffraction des rayons $\mathrm{X}$ par un échantillon monoatomique). Pour les neutrons les longueurs de diffusion $b$ sont quasiment indépendantes de $q$ et l'on peut intégrer $d \sigma / d \Omega$ de manière directe pour obtenir la section efficace totale $\sigma$ de l'échantillon qui est alors la somme de 2 termes:

$$
\sigma=\int_{4 \pi} \frac{d \sigma}{d \Omega} d \Omega=\sigma_{\mathrm{coh}}+\sigma_{\mathrm{incoh}}=4 \pi N b_{\mathrm{coh}}^{2}+4 \pi N b_{\mathrm{incoh}}^{2}=4 \pi N \overline{b^{2}}
$$

où $d \Omega=2 d \phi \sin (2 \theta) d \theta=(\lambda / 2 \pi)^{2} d \phi q d q$ et l'intégrale pour la partie cohérente n'est précise que pour des longueurs d'ondes $\lambda$ petites par rapport à la distance interatomique. Pour comprendre cela, pensez au cas contraire, où $\lambda$ est assez grand pour que le premier pic de Bragg (diffraction par un poudre) se trouve à un angle $2 \theta>180^{\circ}-$ il n'y aura alors aucune diffraction cohérente (comme pour un filtre de $\mathrm{Be}$ en neutronique). Effectivement, on définit $\sigma_{\mathrm{coh}} \equiv \sigma_{\mathrm{coh}, \lambda}(\lambda \rightarrow 0)=4 \pi N \bar{b}^{2}$ pour les neutrons. Une compilation des valeurs de $b_{\mathrm{coh}}, b_{\text {incoh }}, \sigma_{\mathrm{coh}}$ et $\sigma_{\text {incoh }}$ pour la plupart des isotopes connus est donnée dans la Ref. [14].

Il est souvent plus commode de décomposer $d \sigma / d \Omega$ de manière legèrement différente: une contribution "distincte" (relative à l'interférence entre les atomes) et une contribution "self" (relative aux atomes isolés):

$$
\begin{aligned}
\frac{1}{N}\left[\frac{d \sigma}{d \Omega}(\mathbf{q})\right] & =\frac{1}{N}\left[\frac{d \sigma}{d \Omega}(\mathbf{q})\right]^{\text {distinct }}+\frac{1}{N}\left[\frac{d \sigma}{d \Omega}(\mathbf{q})\right]^{\text {self }} \\
& =\bar{b}^{2} F(\mathbf{q})+\overline{b^{2}}
\end{aligned}
$$

où

$$
F(\mathbf{q})=\frac{1}{N}\left\langle\sum_{i, j \neq i}^{N} e^{i \mathbf{q} \cdot \mathbf{r}_{i j}}\right\rangle=S(\mathbf{q})-1
$$

est la fonction d'interférence (i.e. pour des sites atomiques différents). Notons les valeurs limites $F(q \rightarrow \infty)=0$ et $S(q \rightarrow \infty)=1$, et que $S(\mathbf{q}) \geq 0$ toujours, mais $F(\mathbf{q})$ peut être de valeur négative. La Fig. 1 montre des exemples de $S(q)$ pour des systèmes différents.

Puisqu'on considère toujours l'approximation statique, $S(\mathbf{q})$ est l'intégrale du facteur de structure dynamique $S(\mathbf{q}, \omega)$ sur tous les échanges d'énergie par diffusion inélastique entre le neutron ou rayon $\mathrm{X}$ et l'échantillon:

$$
S(\mathbf{q})=\int_{-\infty}^{+\infty} d \omega S(\mathbf{q}, \omega)
$$



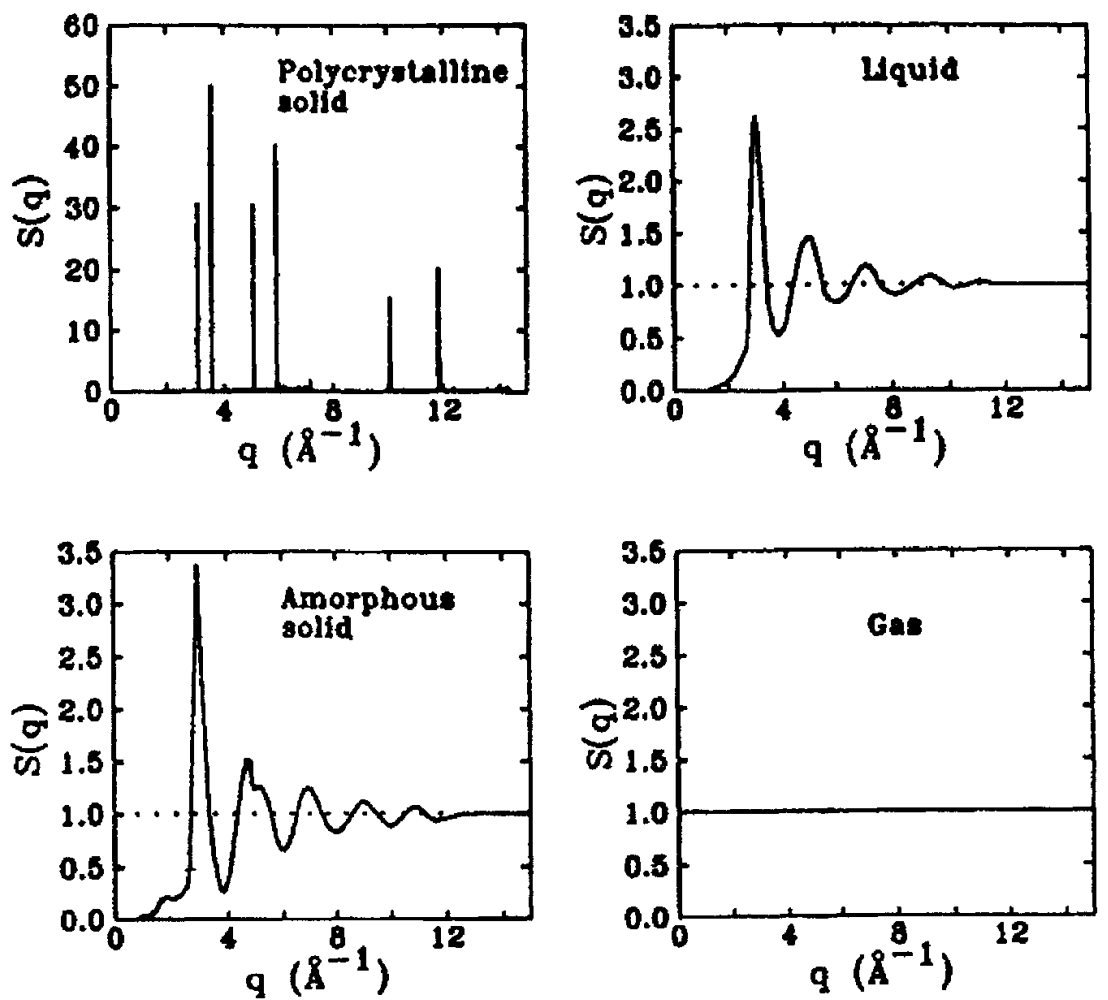

Figure 1. Facteurs de structure $S(q)$ pour des matériaux différents. Pour nous, un "matériau amorphe" veut dire un verre. Le $S(q)$ montré pour un gaz est dans le cas $\rho \rightarrow 0$.

En réalité la présence d'un échange maximal d'énergie implique un temps de "snapshot" $t_{\min } \sim \omega_{\max }^{-1}$ qui pour le cas de diffraction des neutrons tombe dans la fourchette de $10^{-15}-10^{-13} \mathrm{~s}$.

La structure d'un échantillon (toujours monoatomique ici) peut être décrite dans l'espace réel en termes de sa fonction de distribution de paires $g(\mathbf{r})$ qui est relative à la probabilité de trouver un atome à une position relative $\mathbf{r}$ par rapport à un atome de référence à l'origine. Les fonctions $S(\mathbf{q})$ et $g(\mathbf{r})$ sont liées par transformation Fourier:

$$
\begin{aligned}
& S(\mathbf{q})-1=\rho_{0} \int[g(\mathbf{r})-1] e^{i \mathbf{q} \cdot \mathbf{r}} d \mathbf{r} \\
& g(\mathbf{r})-1=\frac{1}{\rho_{0}(2 \pi)^{3}} \int[S(\mathbf{q})-1] e^{-i \mathbf{q} \cdot \mathbf{r}} d \mathbf{q},
\end{aligned}
$$

où $\rho_{0}$ est la densité d'atomes et l'on voit que $g(\mathbf{r})$ est sans dimension, comme l'est $S(\mathbf{q})$. Dans le cas d'un échantillon liquide ou vitreux pour lesquels la structure moyenne est 
isotrope, seulement les normes $r=|\mathbf{r}|$ et $q=|\mathbf{q}|$ sont pertinentes et l'on peut remplacer les $\exp (i \mathbf{q} \cdot \mathbf{r})$ par des $\sin (q r) /(q r)$ et ainsi simplifier l'expression pour le facteur de structure (statique):

$$
S(q)=\frac{1}{N}\left\langle\sum_{i, j}^{N} \frac{\sin \left(q r_{i j}\right)}{\left(q r_{i j}\right)}\right\rangle=F(q)+1,
$$

et les expressions pour la transformation Fourier en prenant également $\left(d q 4 \pi q^{2}\right)$ et ( $\left.d r 4 \pi r^{2}\right)$ pour les éléments de volume d'intégration:

$$
S(q)-1=\frac{4 \pi \rho_{0}}{q} \int_{0}^{\infty} r[g(r)-1] \sin (q r) d r
$$

et

$$
g(r)-1=\frac{1}{2 \pi^{2} r \rho_{0}} \int_{0}^{\infty} q[S(q)-1] \sin (q r) d q,
$$

d'où $S(q \rightarrow \infty)=1$ et $g(r \rightarrow \infty)=1$, et en pratique l'intégration est limitée à $q_{\max }$. La diffraction devient alors conique (i.e. Debye-Scherrer). C'est par l'emploi de l'Eq. (15) qu'une mésure de diffraction $S(q)$ sur un verre/liquide se traduit en information structurale $g(r)$ qui peut être comparée à des prévisions théoriques ou à des résultats des simulations. La Fig. 2 montre les aspects différents du facteur de structure $S(q)$ d'un liquide ou verre. Pour un échantillon monoatomique, le premier grand pic en $S(q)$ est d'habitude relatif à une distance moyenne $d \approx 2 \pi / q_{\text {pic }}$ entre atomes voisins. Pour un système polyatomique, ce pic (le "first sharp diffraction peak" ou "FSDP") est relatif à la distance moyenne interatomique ou intermoléculaire, selon le cas.

La Fig. 3(a) représente une fonction de distribution de paires $g(r)$ typique pour un liquide ou verre. La présence d'oscillations en $g(r)$ pour de petites valeurs de $r$ témoigne de l'imperfection des données $S(q)$, celle-ci étant due par example à une faible statistique de comptage, ou à une gamme de $q$ trop limitée - plus le transfert de moment maximal $q_{\max }$ est grande, plus la structure à courte échelle dans l'espace réel est précisée. Par exemple, les importants $q_{\max }=17 \AA^{-1}$ et $24 \AA^{-1}$ (pour $\lambda=0.7 \AA$ et $0.5 \AA$ ) disponibles sur le diffractomètre $\mathrm{D} 4$ à l'Institut Laue-Langevin (ILL) permettent une bonne résolution à l'échelle atomique [15].

On voit par l'Eq. (14) que dans la limite de dilution infinie $\rho_{0} \rightarrow 0$ (i.e. un gaz parfait), $S(q)=1$ n'a pas de structure en $q$ (voir encore la Fig. 1 pour un gaz parfait), ce qui est logique car la seule corrélation entre les positions des atomes devient l'autocorrélation d'un atome avec lui-même.

On définit aussi la fonction de corrélation de paires $G(r)$, appelée aussi la fonction de densité $D(r)$, par

$$
G(r)=D(r)=4 \pi r \rho_{0}[g(r)-1],
$$

ainsi que la fonction de distribution radiale $\operatorname{RDF}(r)$ par

$$
\operatorname{RDF}(r)=4 \pi r^{2} \rho_{0} g(r) .
$$



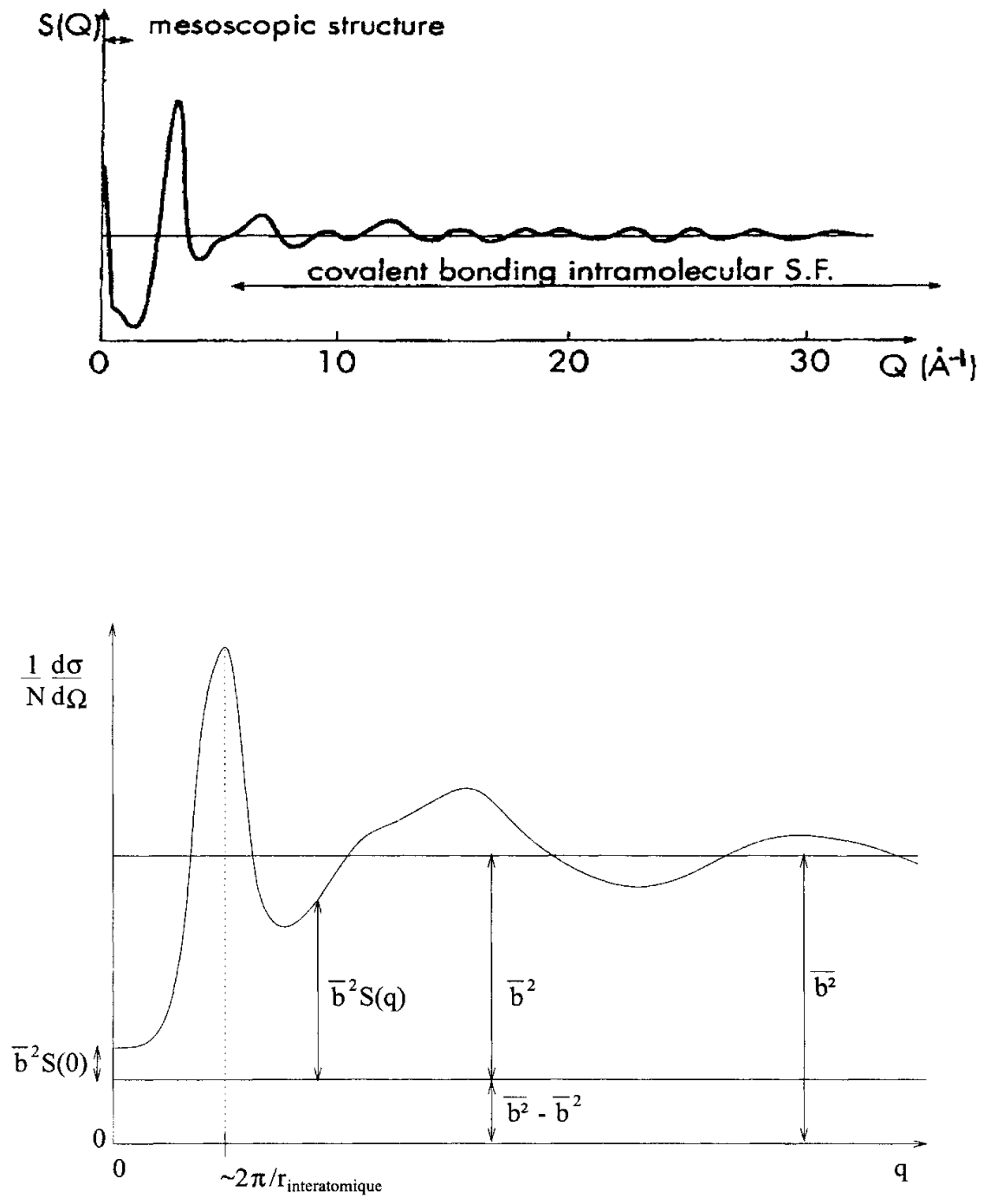

Figure 2. (haut) Exemple de facteur de structure $S(q)$ d'un liquide ou verre indiquant les régions les plus sensibles à différents aspects de la structure. La position du premier pic est relative à l'échelle interatomique ou intermoléculaire. D'après Chieux (1993). (bas) Les intensités caractéristiques de la section efficace par atome $(1 / N) d \sigma / d \Omega=\bar{b}^{2} S(q)$ d'un liquide monoatomique simple. 

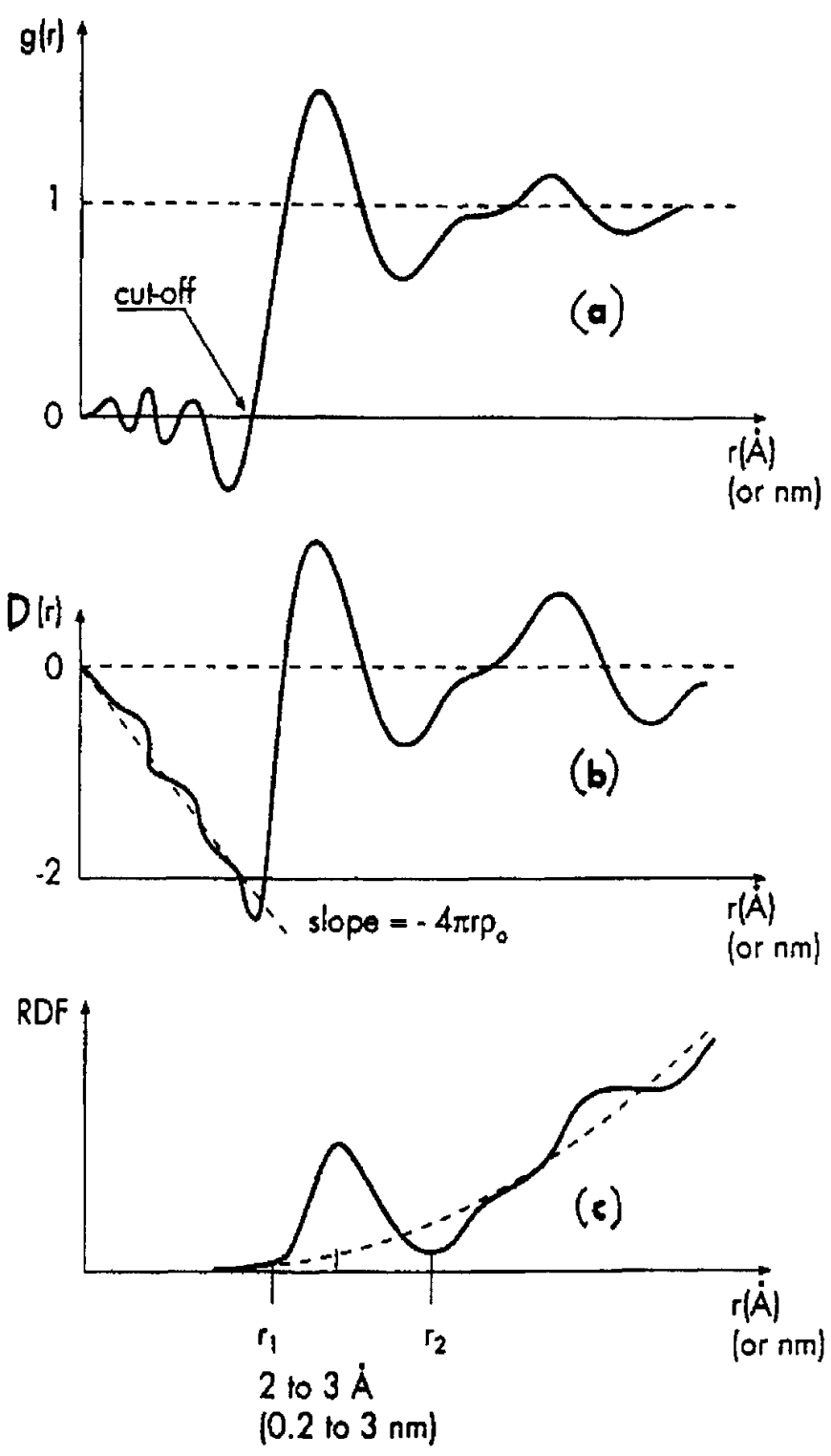

Figure 3. Fonctions de structure dans l'espace réel pour un liquide/verre: (a) fonction de distribution de paires $g(r)$, (b) fonction de corrélation de paires $G(r)$ ou fonction de densité $D(r)$, (c) fonction de distribution radiale $\operatorname{RDF}(r)$. D'après Chieux (1993). 
Le dérivé de $D(r)$ pour de petites valeurs de $r$ est proportionnel à $\rho_{0}$ (la Fig. 3(b)), alors que la fonction $\operatorname{RDF}(r)$ permet de déterminer par intégration le nombre de coordination $\bar{n}$ des atomes voisins, c'est à dire le nombre moyen d'atomes dans une "couche" sphérique autour d'un atome donné:

$$
\bar{n}=\int_{r_{1}}^{r_{2}} \operatorname{RDF}(r) d r=4 \pi \rho_{0} \int_{r_{1}}^{r_{2}} g(r) r^{2} d r
$$

où $r_{1}$ et $r_{2}$ sont des rayons consecutifs qui donnent un minimum en $g(r)$ (la Fig. 3(c)).

Dans un fluide, la probabilité de trouver une certaine paire d'atomes à une distance $r$ à un moment donné est évidemment liée au potentiel de paires $u(r)$ entre eux. Le fluide étant un système ergodique, une moyenne de l'ensemble des configurations possibles à un moment donné égale une moyenne temporelle pour un membre donné (i.e. une paire d'atomes) de cet ensemble. Ceci permet, à partir d'une mesure de diffraction de $S(q)$ d'un liquide (ou d'un verre considéré comme un liquide gelé en temps) qui sonde une moyenne d'ensemble par l'Eq. (1), de déterminer certains paramètres qui appartiennent normalement à la description dynamique du système, tels que la vitesse du son ou bien le potentiel de paires [16]. On voit donc qu'un système désordonné peut parfois apporter plus d'informations qu'un système ordonné lors d'une mesure de la structure statique. En effet, l'ensemble des configurations atomiques présentes au travers du volume d'un échantillon liquide ou vitreux "explore" le potentiel de paires d'une manière analogue à celle effectuée par des vibrations et diffusions atomiques.

À ce propos, la limite thermodynamique $S(q \rightarrow 0)$ est relative aux propriétés de masse de l'échantillon (monoatomique) pour un système désordonné (mais non pour un cristal):

$$
S(0)=\rho_{0} \chi_{T} k_{B} T,
$$

ò̀ $\chi_{T}$ est la compressibilité isothermique. Ce résultat est intuitif car la présence de fluctuations de densité à travers le volume de l'échantillon donne lieu à une diffusion aux petits angles voire à $q=0$, et implique également une compressibilité car les régions moins denses pourraient devenir, a priori, aussi denses que les régions les plus denses. Dans le cas d'un gaz parfait, cette expression de $S(0)$ se réduit à l'équation d'état: $P=\rho_{0} k_{B} T$.

\subsection{Le cas de plusieurs espèces atomiques (i.e. polyatomique)}

En derivant les équations (7) et (9) pour un système monoatomique, on a supposé qu'il n'y a pas de corrélation entre les longueurs de diffusion et les positions des atomes. Ceci a permis d'extraire un facteur de $\bar{b}^{2}$ puis d'interpreter le résultant $S(q)$ sans dimension et sa transformation Fourier $g(r)$ en termes physiques assez simples, par exemple la valeur limite $S(q \rightarrow 0)$ était proportionelle à la compressibilité isothermique.

Dans le cas d'un système polyatomique, les propriétés chimiques différentes des espèces atomiques différentes donnent évidemment lieu à une corrélation entre les longueurs de diffusion et les positions des atomes qui est inéchappable. On est donc contraint de la respecter et de définir des facteurs de structure partiels qui tiennent compte des espèces atomiques présentes dans l'échantillon. Ceux-ci permettront juste- 
ment de décrire la structure en fonction des espèces atomiques (e.g. l'arrangement des atomes d'oxygène autour d'un site occupé par un atome d'hydrogène).

La généralisation de l'Eq. (9) à un système isotrope de $n$ espèces atomiques (i.e. présentant $n$ nombres atomiques $Z$ différents) est directe et se décompose aussi en parties "distincte" et "self":

$$
\frac{1}{N}\left[\frac{d \sigma}{d \Omega}(q)\right]=F_{\mathrm{T}}(q)+\sum_{\alpha}^{n} c_{\alpha} \overline{b^{2}} \alpha,
$$

où la fonction d'interférence totale $F_{\mathrm{T}}(q)$ entre tous les atomes est donnée par une somme pondérée des fonctions d'interférences partielles $F_{\alpha \beta}(q)$ des atomes d'espèce $\alpha$ avec ceux d'espèce $\beta$ :

$$
F_{\mathrm{T}}(q)=\sum_{\alpha, \beta}^{n} c_{\alpha} c_{\beta} \bar{b}_{\alpha} \bar{b}_{\beta}^{*} F_{\alpha \beta}(q),
$$

pour laquelle $c_{\alpha}$ est la concentration de l'espèce $\alpha$ et $\sum_{\alpha}^{n} c_{\alpha}=1$. Notons que les barres de moyenne $(\bar{b})$ sont superflues pour les rayons $X$ et que les * ne sont que rarement nécessaires pour les neutrons. Notons également que la somme pondérée donne une quantité qui est forcément réelle car, par exemple, $\bar{b}_{1} \bar{b}_{2}^{*}+\bar{b}_{2} \bar{b}_{1}^{*}=2 \Re\left(\bar{b}_{1} \bar{b}_{2}^{*}\right)=2 \Re\left(\bar{b}_{2} \bar{b}_{1}^{*}\right)$ (bien que non égal à $2\left|\bar{b}_{1}\right|\left|\vec{b}_{2}\right|$ ni à $\left.2 \Re\left(\bar{b}_{1}\right) \Re\left(\bar{b}_{2}\right)\right)$. Finalement on voit que les $F_{\alpha \beta}(q)$ sont sans dimension mais que $F_{\mathrm{T}}(q)$ a les mêmes unités (barns/str) que $d \sigma / d \Omega$.

De manière analogue au cas d'un échantillon monoatomique, la fonction d'interférence partielle $F_{\alpha \beta}(q)$ représente la somme des produits d'amplitudes de diffusion provenant des sites distincts $i$ (parmi les atomes $\alpha$ ) et $j$ (parmi les atomes $\beta$ ). En comparant l'équation (1) avec les équations (20) et (21), on voit que les $F_{\alpha \beta}(q)$ sont donnés par:

$$
\begin{aligned}
F_{\alpha \beta}(q)=F_{\beta \alpha}(q) & =\frac{1}{c_{\alpha} c_{\beta} N \Re\left(\bar{b}_{\alpha} \bar{b}_{\beta}^{*}\right)} \Re\left(\bar{b}_{\alpha} \bar{b}_{\beta}^{*}\left\langle\sum_{i, j \neq i}^{N_{\alpha}, N_{\beta}} e^{i \mathbf{q} \cdot \mathbf{r}_{i j}}\right\rangle\right) \\
& =\frac{1}{c_{\alpha} c_{\beta} N}\left\langle\sum_{i, j \neq i}^{N_{\alpha}, N_{\beta}} \frac{\sin \left(q r_{i j}\right)}{\left(q r_{i j}\right)}\right\rangle,
\end{aligned}
$$

où $N_{\alpha}=c_{\alpha} N$ est le nombre d'atomes $\alpha$ et évidemment l'équivalence des sites $i=j$ n'est possible que pour un même espèce atomique (le cas $\alpha=\beta$ ). Notons d'ailleurs que $F_{\alpha \beta}(q \rightarrow \infty)=0$ même pour $\alpha=\beta$. Les facteurs de structure partiels $S_{\alpha \beta}(q)=S_{\beta \alpha}(q)$ sont définis comme

$$
S_{\alpha \beta}(q)=F_{\alpha \beta}(q)+1
$$

et convergent à 1 pour $q \rightarrow \infty$ comme pour le cas monoatomique. Les $S_{\alpha \beta}(q)$ sont liées par transformation Fourier aux fonctions de distribution de paires partielles $g_{\alpha \beta}(r)$ :

$$
\begin{aligned}
& S_{\alpha \beta}(q)-1=\frac{4 \pi \rho_{0}}{q} \int_{0}^{\infty} r\left[g_{\alpha \beta}(r)-1\right] \sin (q r) d r \\
& g_{\alpha \beta}(r)-1=\frac{1}{2 \pi^{2} r \rho_{0}} \int_{0}^{\infty} q\left[S_{\alpha \beta}(q)-1\right] \sin (q r) d q
\end{aligned}
$$


où $\rho_{0}$ est toujours la densité totale des atomes. Les $g_{\alpha \beta}(r)$ sont relatives à la probabilité de trouver un atome $\beta$ à une distance $r$ d'un atome $\alpha$ à l'origine. Plus précisement, le nombre de coordination partiel (ici le nombre moyen d'atomes $\beta$ dans une couche sphérique autour d'un atome $\alpha$ ) est donné par intégration d'une fonction de distribution radiale partielle:

$$
\bar{n}_{\alpha}^{\beta}=4 \pi \rho_{0} c_{\beta} \int_{r_{1}}^{r_{2}} g_{\alpha \beta}(r) r^{2} d r .
$$

Le facteur de structure total $S_{\mathrm{T}}(q)$ défini comme

$$
S_{\mathrm{T}}(q)=\sum_{\alpha, \beta}^{n} c_{\alpha} c_{\beta} \bar{b}_{\alpha} \bar{b}_{\beta}^{*} S_{\alpha \beta}(q)=F_{\mathrm{T}}(q)+\sum_{\alpha, \beta}^{n} c_{\alpha} c_{\beta} \bar{b}_{\alpha} \bar{b}_{\beta}^{*}
$$

converge ̀̀ $\sum_{\alpha, \beta}^{n} c_{\alpha} c_{\beta} \bar{b}_{\alpha} \bar{b}_{\beta}^{*}=\left|\sum_{\alpha}^{n} c_{\alpha} \bar{b}_{\alpha}\right|^{2}$. En notant que

$$
\left|\sum_{\alpha}^{n} c_{\alpha} \bar{b}_{\alpha}\right|^{2}=\bar{b}^{2} \quad \text { et } \quad \sum_{\alpha}^{n} c_{\alpha} \bar{b}_{\alpha}{ }_{\alpha}=\overline{b^{2}}
$$

sont toujours les bonnes moyennes à travers l'échantillon, on peut écrire donc pour un échantillon polyatomique:

$$
\begin{aligned}
\frac{1}{N}\left[\frac{d \sigma}{d \Omega}(q)\right] & =S_{\mathrm{T}}(q)+\left(\overline{b^{2}}-\bar{b}^{2}\right) \\
& =F_{\mathrm{T}}(q)+\overline{b^{2}}
\end{aligned}
$$

où l'on voit que la contribution incohérente est toujours la variance totale de la distribution de $b$, comme dans l'Eq. (7), et ici elle est évidemment non nulle pour les rayons $\mathrm{X}$ car il y a plusieurs $Z$ dans l'échantillon. Ce résultat de l'Eq. (29) est intuitif parce que pour la diffusion incohérente le neutron ou rayon $X$ "voit" seulement la distribution de $b$ dans l'échantillon et non la structure dépendant de la chimie (et donc de $Z$ ). Si l'on essaie de récupérer la limite thermodynamique de l'Eq. (19) dans le cas d'un échantillon polyatomique, il faut normaliser le facteur de structure de la manière suivante [3]:

$$
S(q)=\frac{S_{\mathrm{T}}(q)}{\left|\sum_{\alpha}^{n} c_{\alpha} \bar{b}_{\alpha}\right|^{2}}=\frac{\sum_{\alpha, \beta}^{n} c_{\alpha} c_{\beta} \bar{b}_{\alpha} \bar{b}_{\beta}^{*}\left(S_{\alpha \beta}(q)-1\right)}{\left|\sum_{\alpha}^{n} c_{\alpha} \bar{b}_{\alpha}\right|^{2}}+1,
$$

pour lequel $S(q \rightarrow \infty)=1$ mais $S(0) \geq \rho_{0} \chi_{T} k_{B} T$ car non seulement les fuctuations en densité contribuent à l'intensité à $q \rightarrow 0$ mais également les fluctuations en concentration pour un système polyatomique. Notons que la décomposition de $S_{\mathrm{T}}(q)$ et $F_{\mathrm{T}}(q)$ par les équations (27) et (21) en $S_{\alpha \beta}(q)$ et $F_{\alpha \beta}(q)$ est celle proposée par Faber et Ziman [17], et qu'il existe d'autres conventions pour définir des facteurs de structure partiels comme celle de Bhatia et Thornton [18], cette dernière étant justement plus commode pour considérer la limite thermodynamique $q \rightarrow 0$.

Le but est donc d'accéder aux facteurs de structures partiels puis aux fonctions de distribution de paires partiels par transformation Fourier, celles-ci pouvant être comparées de manière quantitative aux modèles théoriques et/ou aux simulations de 
la structure du système. Les deux sections suivantes décrivront chacune les "recettes" pour déterminer les facteurs de structures partiels par des techniques spéciales de diffraction des neutrons et des rayons $\mathrm{X}$, respectivement, $\mathrm{y}$ compris une briève discussion des erreurs systématiques inhérentes à chaque technique. La dernière section discutera la combinaison des techniques de diffraction des neutrons et des rayons $\mathrm{X}$.

\section{DIFFRACTION DES NEUTRONS AVEC SUBSTITUTION ISOTO- PIQUE (NDIS)}

\subsection{Erreurs systématiques dans les mesures de diffraction des neutrons}

Pour obtenir $d \sigma / d \Omega$ de l'échantillon dans une expérience de diffraction des neutrons, il faut naturellement soustraire les contributions à l'intensité provenant du container de l'échantillon, de l'environnement de l'échantillon (e.g. du four, du cryostat), et du bruit de fond (neutronique ainsi qu'électronique). Ceci nécessite des mesures de diffraction pour le container vide, le four vide, etc. La normalisation de $d \sigma / d \Omega$ s'effectue ensuite soit par rapport à l'intensité de diffraction d'un échantillon de $\sigma_{\text {self }}$ connu (e.g. vanadium), soit à partir des limites $S(0)$ et $S(\infty)$ mentionnées précédemment. Il faut également tenir compte des éventuelles corrections à $d \sigma / d \Omega$ dues à l'attenuation, à la diffusion multiple et à la diffusion inélastique.

Les corrections d'attenuation (résultant de l'absorption et de la diffusion des quanta) ont été traitées par Paalman et Pings [19] pour le cas d'un échantillon de géométrie cylindrique entouré par un container annulaire. Ils présentent des coefficients, dépendant de l'angle de diffraction, qui permettent de soustraire correctement le diffractogramme du container vide en tenant compte de l'attenuation du container et de l'échantillon. Notons que les coefficients d'attenuation pour les neutrons sont généralement beaucoup plus faible que ceux pour les rayons $\mathrm{X}$ à une longueur d'onde donnée.

Les résultats de Paalman et Pings présupposent que chaque neutron qui contribue au diffractogramme n'est diffusé qu'une seule fois, d'où la question de la diffusion multiple. Par définition, $d \sigma / d \Omega$ est proportionnel au nombre total d'événements de diffusion des neutrons dans l'échantillon. Pourtant, un neutron peut diffuser plusieurs fois avant d'être compté (seulement une fois) par le détecteur sous un angle modifié par le premier événement (et les suivants). La diffusion multiple mène par conséquent à une sousestimation (traitée effectivement par les corrections d'attenuation), ainsi qu'à une distortion, de $d \sigma / d \Omega$. Pour un échantillon qui diffuse moins que $10 \%$ du faisceau, la distortion de $d \sigma / d \Omega$ est faible. Les corrections pour une diffusion multiple importante dépendent naturellement de la géométrie de l'échantillon et de son environnement illuminé par le faisceau. La complexité des solutions analytiques [20] pour container+échantillon encourage l'exploitation de simulations de type Monte Carlo adaptées à la géométrie exacte de l'expérience [21].

Pour les expériences de diffraction sur des échantillons (surtout des échantillons fluides) contenant des éléments de faible masse atomique ( $M \lesssim 20 \mathrm{amu})$, l'approximation 

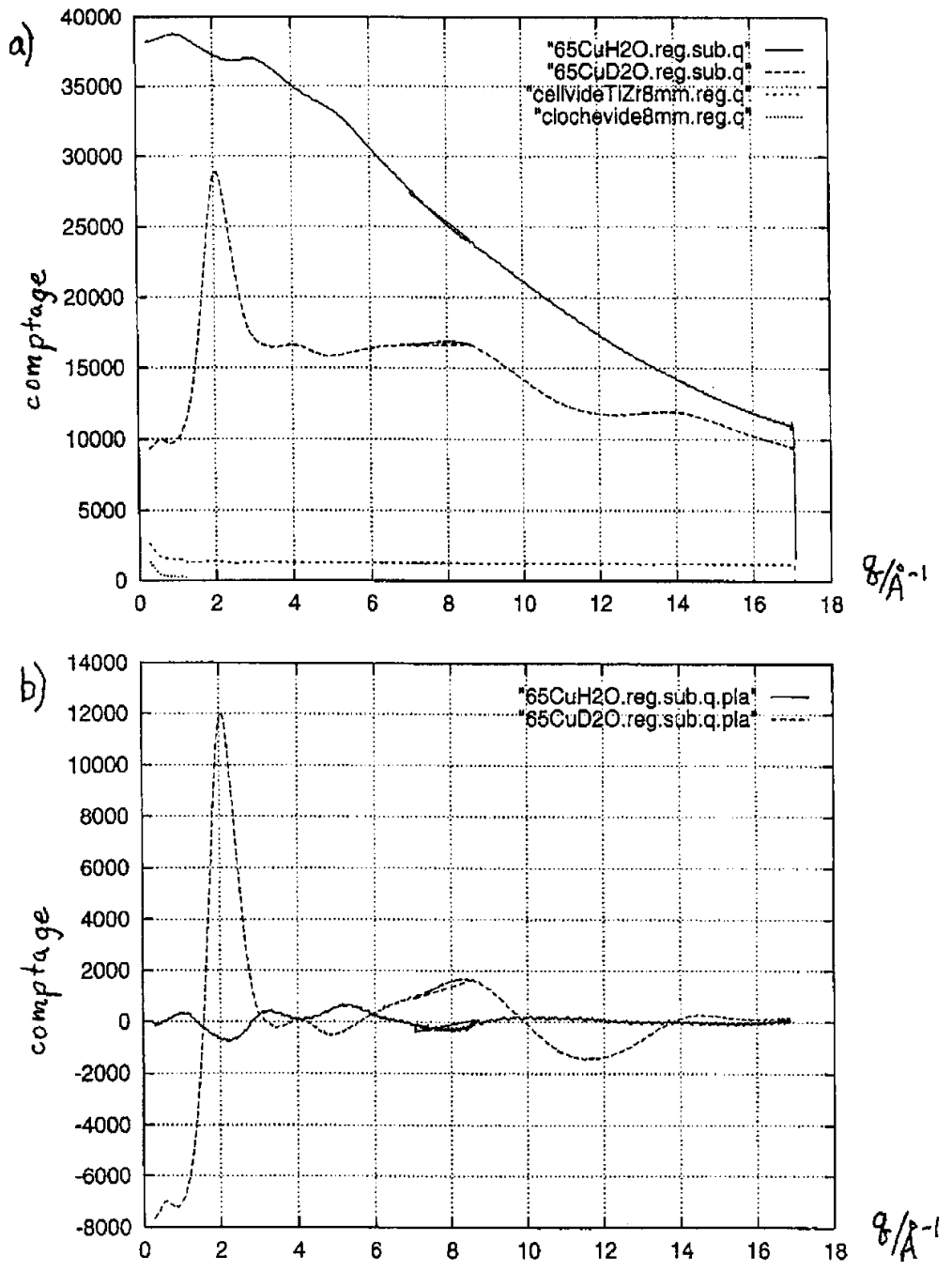

Figure 4. (a) Exemples des données $\propto d \sigma / d \Omega$ d'un diffractomètre de neutrons pour un échantillon liquide hydrogéné (courbe du haut), un échantillon liquide deutéré (courbe du milieu), ainsi que les contributions (déjà soustraites) du container en TiZr et du bruit de fond (courbes du bas), (b) Les données $\propto F_{\mathrm{T}}(q)$ pour les mêmes échantillons après soustraction du "self" et la correction d'inélasticité. 
statique $\left(E_{\text {fin }} \approx E_{\text {inc }}\right)$ n'est plus valable car le transfert d'énérgie entre les neutrons et l'échantillon devient important. De plus, l'intégration de l'Eq. (11) est faite par le détecteur à $2 \theta$ constant et non à $q$ constant. Finalement, l'efficacité de détection dépend généralement de l'énergie du neutron capté. Ce problème composite de la correction de diffusion inélastique a été traité par Placzek [22] dont les résultats peuvent être écrits de manière simplifiée pour la majorité des cas [23]. En première approximation, seule l'intensité "self" est affectée:

$$
\left[\frac{d \sigma}{d \Omega}(q)\right]_{\text {mesure }}^{\text {self }}=\left[\frac{d \sigma}{d \Omega}(q)\right]_{\text {theorie }}^{\text {self }}[1+P(q)],
$$

où $P(q)$ est une expansion en puissances de $q^{2}$ et des rapports $m / M_{\alpha}$ de la masse du neutron sur celles des atomes dans l'échantillon. En pratique, il s'agit de soustraire un polynome en $q^{2}$ de $d \sigma / d \Omega$ pour obtenir directement $F(q)$ après normalisation (I'Eq. (9)). Pour des échantillons contenant de l'hydrogène, l'expansion en puissances de $m / M_{\mathrm{H}}=1$ de l'Eq. (31) ne se tient plus. De plus, l'hydrogène donne lieu a une forte diffusion incohérente. Une bonne correction d'inélasticité de $S(q)$ pour des atomes $\mathrm{H}$ nécessiterait la connaissance de $S(q, \omega)$. Cependant, en pratique l'affinement de $d \sigma / d \Omega$ en fonction de $q$ (et non de 20) par une fonction pseudovoigt (combinaison linéaire d'une lorentzienne et d'une gaussienne normalisées à la même largeur à mi-hauteur) se rapproche très bien de l'intensité "self" d'un échantillon contenant de l'hydrogène.

Dans une mesure employant une source de réacteur (i.e. monochromatique), $[d \sigma / d \Omega]_{\text {self }}$ diminue avec $q$ de manière dépendante de $\lambda$ pour approcher une valeur asymptote. L'effet Placzek est plus important pour les neutrons d'énergie incidente $E_{\text {inc }}=h^{2} /\left(2 m \lambda^{2}\right)$ faible parce que celle-ci est plus comparable à l'echange $\hbar \omega$ avec l'échantillon. On pourrait penser à cette valeur asymptote comme $N \bar{b}^{2}$ free au lieu de $N \bar{b}^{2}$ bound car plus le transfert de quantité du mouvement $\hbar \mathbf{q}$ est grand, plus le noyau est bousculé donc agissant de manière de plus en plus "libre". À se rappeler que $b_{\text {free }}=b_{\text {bound }}[A /(A+1)]^{2}$ ou $A$ est le poids atomique [24].

Cela donne une façon intuitive de comprendre pourquoi la partie distincte $F_{\mathrm{T}}(q)$ de l'Eq. (21), qui résulte des intérferences entre des atomes distincts, est a priori (et heureusement!) peu affectée par l'inélasticité. Comme la diffusion "self" est isotrope, on peut la considérer comme la diffusion par un noyau isolé qui prend tout le "choc" de $\hbar \mathrm{q}$, tandis que pour la diffusion distincte tous les noyaux dans le volume de cohérence "partagent" $\hbar \mathbf{q}$ (disons à parts égales) et donc leurs positions relatives, qui déterminent $F_{\mathrm{T}}(q)$, sont d'habitude peu affectées et de plus, puisque chacun est moins bousculé, l'on devrait prendre toujours $\bar{b}_{\text {bound }}$ pour leurs longueurs de diffusion et non $\bar{b}_{\text {free }}$.

La Fig. 4 montre des exemples des données $d \sigma / d \Omega$ où les contributions du background et la correction d'inélasticité sont prises en compte.

\subsection{Détermination des facteurs de structure partiels par NDIS}

Pour accéder aux facteurs de structures partiels $S_{\alpha \beta}(q)$ (et ainsi aux $g_{\alpha \beta}(r)$ ) à partir des mesures de $S(q)$, on fait appel à la technique de substitution isotopique pour la diffraction des neutrons (NDIS) [1,2,3]. Il s'agit de mesurer $S_{\mathrm{T}}(q)$ ou $F_{\mathrm{T}}(q)$ pour plusieurs échantillons de composition chimique identique (i.e. les mêmes $c_{\alpha}$ ) et de structure iden- 
tique mais ayant des distributions d'isotopes différentes:

$$
F_{\mathrm{T} i}(q)=\sum_{\alpha, \beta}^{n} c_{\alpha} c_{\beta} \bar{b}_{\alpha i} \bar{b}_{\beta i} F_{\alpha \beta}(q)
$$

où $\bar{b}_{\alpha i}$ est la longueur de diffusion moyenne pour le type d'atome $\alpha$ de l'échantillon $i$ (dans cette section on supposera pour simplicité que toutes les longueurs de diffusion ont des valeurs purement réelles - la Ref. [14] donne les $\bar{b}$ pour la plupart des isotopes connus). Les $F_{\mathrm{T} i}(q)$ sont obtenus par l'Eq. (20) après la normalisation et les éventuelles corrections des $d \sigma / d \Omega$ discutées précédemment. Pour déterminer un nombre $m$ de $F_{\alpha \beta}(q)$, il faut un nombre $m$ d'échantillons différents, d'où l'Eq. (32) s'exprime généralement en forme de matrice. Pour un composé de $n=2$ espèces atomiques différents (dénotées $x$ et $y$ ), e.g. pour un alliage binaire, nous avons $m=n(n+1) / 2=3$ facteurs de structure partiels et donc:

$$
\begin{aligned}
{\left[\begin{array}{l}
F_{\mathrm{T} 1}(q) \\
F_{\mathrm{T} 2}(q) \\
F_{\mathrm{T} 3}(q)
\end{array}\right] } & =\left[\begin{array}{lll}
c_{x}^{2} \bar{b}_{x 1}^{2} & c_{y}^{2} \bar{b}_{y 1}^{2} & 2 c_{x} c_{y} \bar{b}_{x 1} \bar{b}_{y 1} \\
c_{x}^{2} \bar{b}_{x 2}^{2} & c_{y}^{2} \bar{b}_{y 2}^{2} & 2 c_{x} c_{y} \bar{b}_{x 2} \bar{b}_{y 2} \\
c_{x}^{2} \bar{b}_{x 3}^{2} & c_{y}^{2} \bar{b}_{y 3}^{2} & 2 c_{x} c_{y} \bar{b}_{x 3} \bar{b}_{y 3}
\end{array}\right]\left[\begin{array}{l}
F_{x x}(q) \\
F_{y y}(q) \\
F_{x y}(q)
\end{array}\right] \\
& =\left[\begin{array}{lll}
a_{11} & a_{12} & a_{13} \\
a_{21} & a_{22} & a_{23} \\
a_{31} & a_{32} & a_{33}
\end{array}\right]\left[\begin{array}{l}
F_{x x}(q) \\
F_{y y}(q) \\
F_{x y}(q)
\end{array}\right]
\end{aligned}
$$

c'est à dire

$$
\left[F_{\mathrm{T} i}(q)\right]=\left[A_{i j}\right]\left[F_{j}(q)\right],
$$

qui peut être inversé pour résoudre les fonctions d'intérference partielles $F_{j}(q)$ :

$$
\left[F_{j}(q)\right]=\left[A_{i j}\right]^{-1}\left[F_{\mathrm{T} i}(q)\right] .
$$

Le déterminant normalisé $|A|_{n}$, calculé après avoir divisé chaque rang $i$ de $\left[A_{i j}\right]$ par $\left(\sum_{j} a_{i j}^{2}\right)^{1 / 2}$, est une mesure du conditionnement de la détermination des $F_{j}(q)$, c'est à dire, le contraste entre les $F_{\mathrm{T} i}(q)$. Bien que $|A|_{n}=1$ soit idéal, les expériences de substitution isotopique donnent généralement de valeurs en dessous de 0.1 , ce qui exige une bonne statistique de comptage pour les données. Grosso modo, la valeur du déterminant est la limite acceptable/faisable dans la précision statistique des $F_{\mathrm{T} i}(q)$ pour pouvoir déterminer les facteurs de structure partiels (e.g. pour un déterminant égal à 0.03 il faut avoir des barres d'erreurs en statistique de comptage qui sont plus petites que $3 \%$ ). De plus, puisque les expériences NDIS durent en général plusieurs jours, il faut une très bonne stabilité des détecteurs et de l'environnement de l'échantillon - la diffractomètre D4 à l'ILL a été mis au point pour réaliser des expériences de substitution isotopique en particulier. Les différences entre les diffractogrammes de $F_{\mathrm{T} i}(q)$ étant parfois d'environ $1 \%$, il faut aussi maîtriser suffisamment bien l'élaboration des 3 échantillons pour assurer que leurs structures soient identiques. 


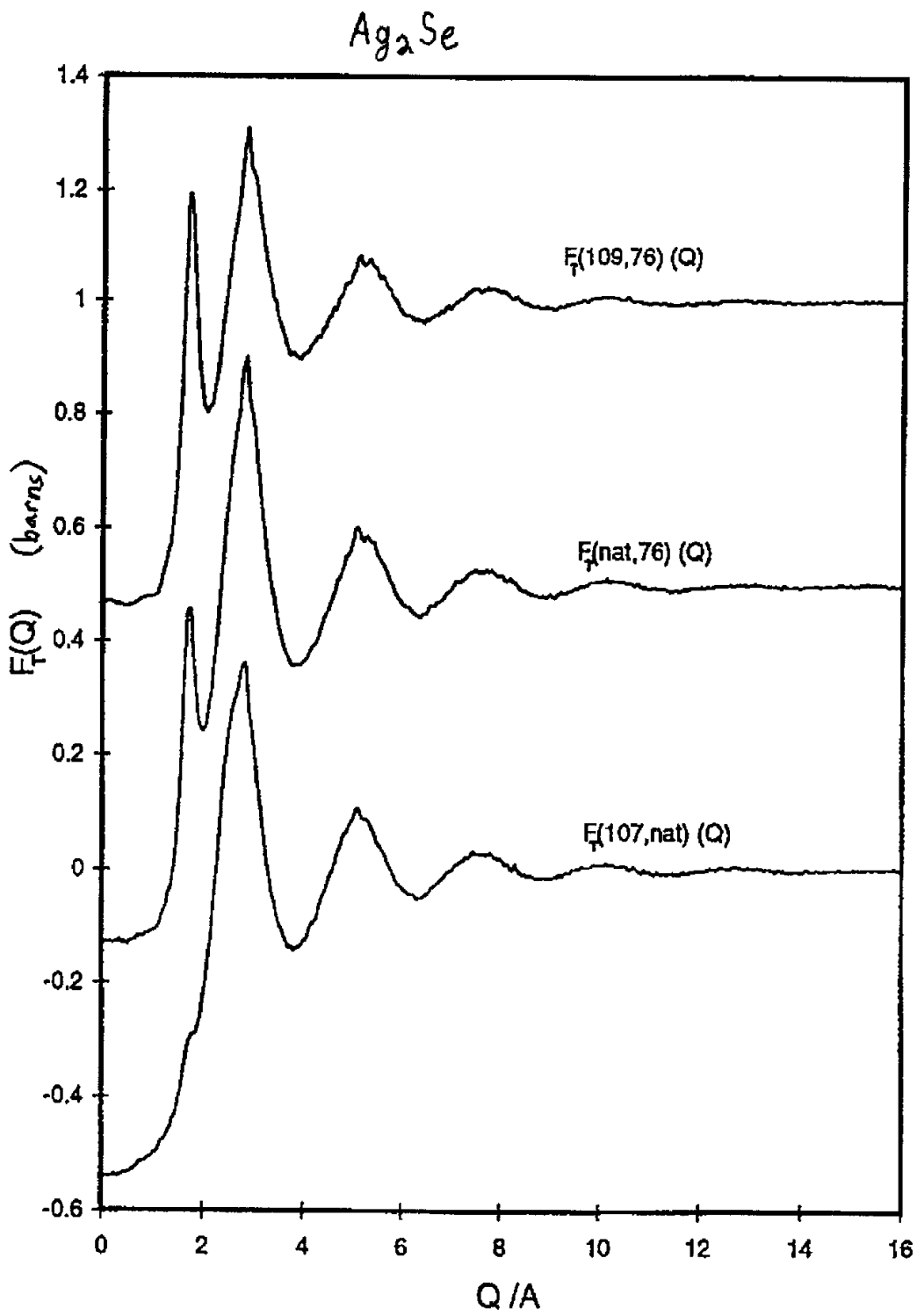

Figure 5. Données d'une expérience de diffraction des neutrons avec substitution isotopique (NDIS): $F_{\mathrm{T}}(q)$ pour 3 échantillons du composé binaire liquide $\mathrm{Ag}_{2}$ Se. Les 2 courbes du haut ont été décalées en ordonnée de 0.5 et de 1.0 . 
La Fig. 5 montre des données $F_{\mathrm{T} i}(q)$ pour 3 échantillons d'un alliage binaire $\left(\mathrm{Ag}_{2} \mathrm{Se}\right)$ isotopiquement distincts: ${ }^{107} \mathrm{Ag}_{2}{ }^{\text {nat }} \mathrm{Se},{ }^{109} \mathrm{Ag}_{2}{ }^{76} \mathrm{Se}$, ${ }^{\text {nat }} \mathrm{Ag}_{2}{ }^{76} \mathrm{Se}$. Dans cette expérience [4], réalisée sur le diffractomètre $\mathrm{D} 4 \mathrm{~b}$, le contraste (i.e. la variation en longueurs de diffusion entre les isotopes) était plutôt bon. On remarque en particulier la variation en intensité du "pre-peak" vers $q=2 \AA^{-1}$. L'équation matricielle (l'Eq. (33)) est donnée par:

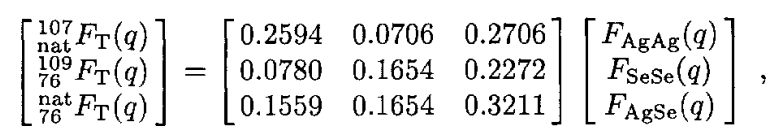

pour lequel $|A|_{n}=0.029$ et dont l'inverse

$$
\left[\begin{array}{c}
F_{\mathrm{AgAg}}(q) \\
F_{\mathrm{SeSe}}(q) \\
F_{\mathrm{AgSe}}(q)
\end{array}\right]=\left[\begin{array}{ccc}
12.17 & 17.31 & -22.50 \\
8.11 & 32.22 & -29.63 \\
-10.09 & -25.00 & 29.30
\end{array}\right]\left[\begin{array}{l}
{ }_{n a t}^{107} F_{\mathrm{T}}(q) \\
109 \\
76
\end{array}\right.
$$

permet de calculer les 3 fonctions d'interférence partielles $F_{\alpha \beta}(q)$ représentées sur la Fig. 6. Les 3 fonctions de distribution de paires partielles $g_{\alpha \beta}(r)$ de la Fig. 7 sont ensuite obtenues par transformation Fourier (l'Eq. (25)). Les $g_{\alpha \beta}(r)$ montrent clairement que la distance du premier voisin pour la paire $\mathrm{Ag}$-Se (i.e. le premier pic) est la plus petite, et qu'il y a une forte anti-corrélation entre les distances correspondant aux paires d'atomes pareils (i.e. $\mathrm{Ag}-\mathrm{Ag}$, Se-Se) et celles correspondant aux paires d'atomes différents ( $\mathrm{Ag}-$ Se). Cette anti-corrélation mène à une annulation efficace du charge moyen autour d'un atome quelconque pour $r \gtrsim 7 \AA$ et est cohérente avec la structure d'un liquide ionique binaire.

Pour un composé contenant plus de 2 espèces atomiques, il est possible de déterminer, à partir de diffractogrammes $F_{\mathrm{T} i}(q)$ relatifs à seulement 3 échantillons de variation isotopique, la fonction d'interférence partielle $F_{x x}(q)$ pour l'un des espèces $x$. Dans ce cas, la distribution isotopique d'uniquement les atomes $x$ est variable entre les 3 échantillons. Par soustraction entre les $F_{\mathrm{T} i}(q)$ relatifs à deux échantillons (voir l'Eq. (21)):

$$
\begin{aligned}
\Delta_{x(1-2)} F_{\mathrm{T}}(q) & \stackrel{\text { def }}{=} F_{\mathrm{T} 1}(q)-F_{\mathrm{T} 2}(q) \\
& =c_{x}^{2}\left(\bar{b}_{x 1}^{2}-\bar{b}_{x 2}^{2}\right) F_{x x}(q)+\sum_{\alpha \neq x}^{n} 2 c_{\alpha} c_{x} \bar{b}_{\alpha}\left(\bar{b}_{x 1}-\bar{b}_{x 2}\right) F_{\alpha x}(q),
\end{aligned}
$$

on obtient une fonction de "première différence" qui ne comprend que des partielles en $x$ (i.e. les $F_{\alpha \alpha}$ ont été éliminés). Une soustraction entre deux autres $F_{\mathrm{T} i}(q)$ mène à un résultat semblable. Finalement, par soustraction de 2 fonctions de première différence (liées à 3 échantillons), on obtient

$$
\begin{aligned}
\Delta_{x}^{2} F_{\mathrm{T}}(q) & \stackrel{\text { def }}{=}\left(\bar{b}_{x 2}-\bar{b}_{x 3}\right)\left[\Delta_{x(1-2)} F_{\mathrm{T}}(q)\right]-\left(\bar{b}_{x 1}-\bar{b}_{x 2}\right)\left[\Delta_{x(2-3)} F_{\mathrm{T}}(q)\right] \\
& =c_{x}^{2}\left[\left(\bar{b}_{x 2}-\bar{b}_{x 3}\right)\left(\bar{b}_{x 1}^{2}-\bar{b}_{x 2}^{2}\right)-\left(\bar{b}_{x 1}-\bar{b}_{x 2}\right)\left(\bar{b}_{x 2}^{2}-\bar{b}_{x 3}^{2}\right)\right] F_{x x}(q),
\end{aligned}
$$

ce qui permet de résoudre $F_{x x}(q)$ par la technique de "double différence" [1]. 


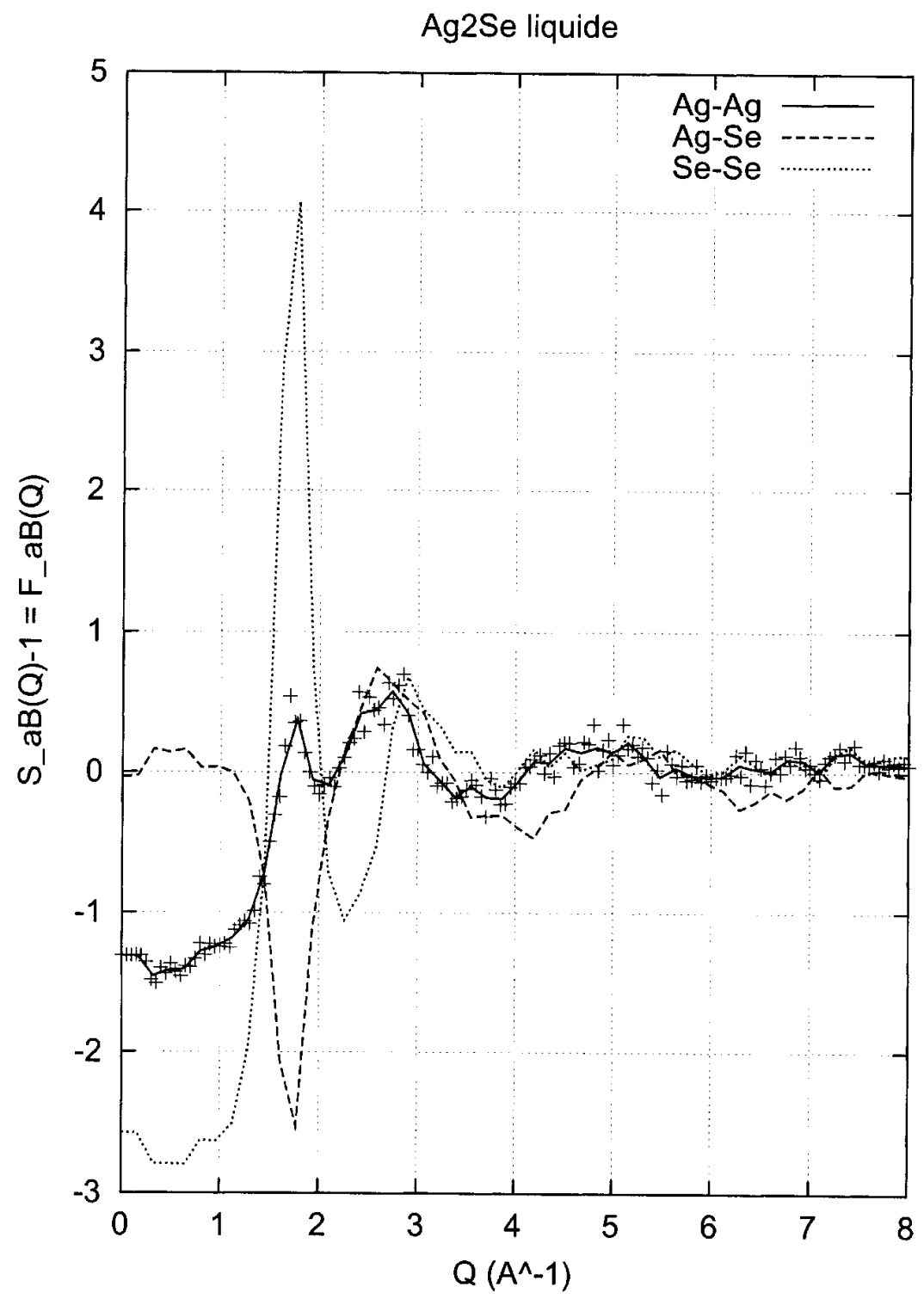

Figure 6. Facteurs de structure partiels $S_{\alpha \beta}(q)-1=F_{\alpha \beta}(q)$ du composé binaire liquide $\mathrm{Ag}_{2}$ Se obtenus par NDIS. 


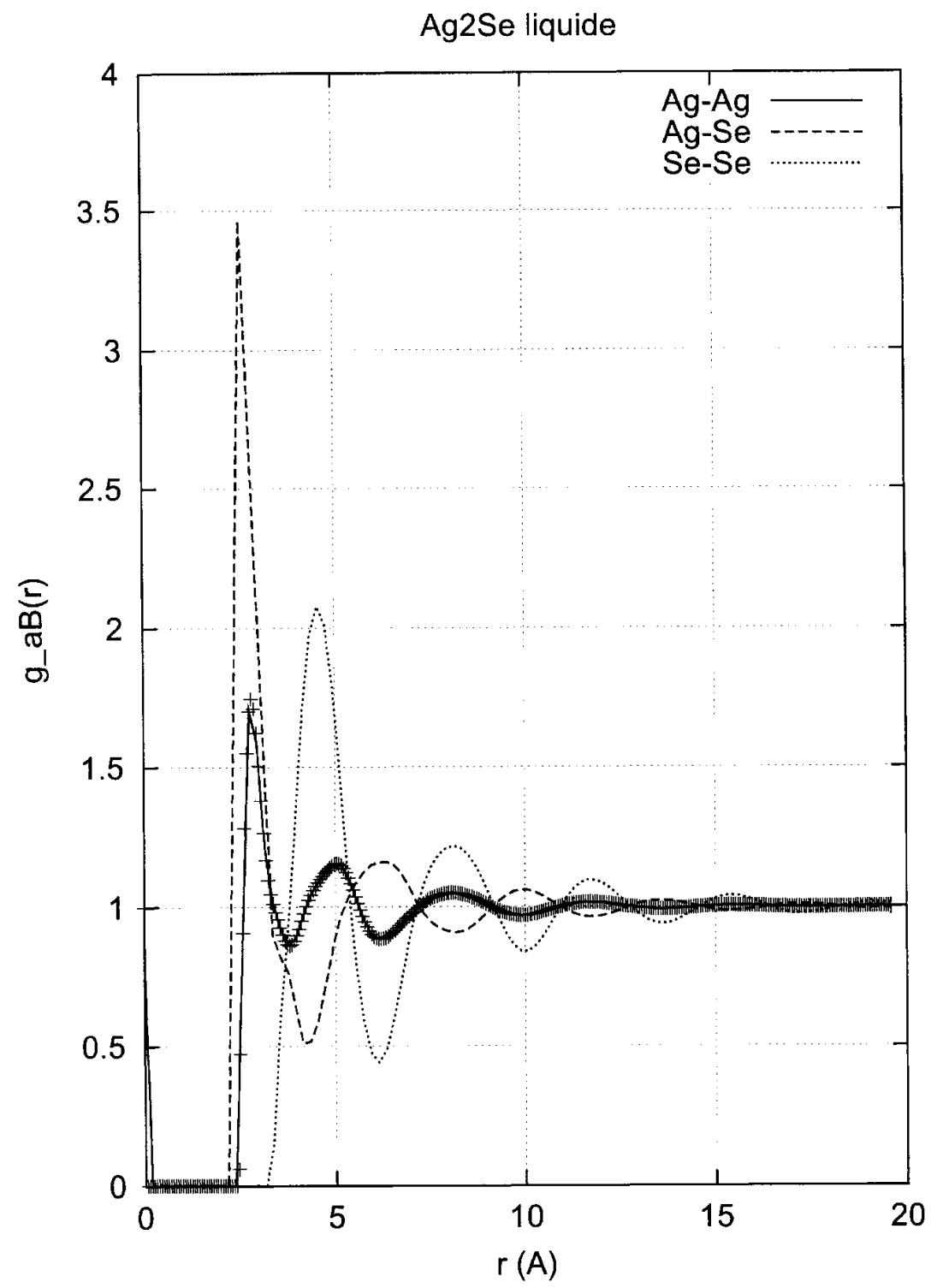

Figure 7. Fonctions de distribution de paires partielles $g_{\alpha \beta}(r)$ du composé binaire liquide $\mathrm{Ag}_{2}$ Se obtenus par NDIS. 


\section{DIFFRACTION ANOMALE DES RAYONS X (AXD)}

\subsection{Erreurs systématiques dans les mesures de diffraction des rayons $X$}

Pour obtenir $d \sigma / d \Omega$ puis $S_{\mathrm{T}}(q)$ d'un échantillon par diffraction des rayons $\mathrm{X}$ il faut naturellement soustraire les contributions à l'intensité provenant du bruit de fond et de l'environnement de l'échantillon, en tenant compte des corrections d'attenuation et de diffusion multiple qui sont plus importantes que dans le cas de la diffraction des neutrons. On verra aussi que les longueurs de diffusion sont dépendantes à la fois en $q$ et en l'énergie incidente $E$.

Les rayons $\mathrm{X}$, qui ont des rapports $E / p$ beaucoup plus importants que ceux des neutrons, n'ont pas le problème d'inélasticité Placzek résultant du recul des noyaux. Cependant, il y a d'autres processus de diffusion non élastique pour les rayons $\mathrm{X}$ tels que la fluorescence, la diffusion Raman résonante, et la diffusion Compton, dont les intensités doivent être soustraites pour obtenir la section efficace "élastique" ou "RayleighThomson" (R.T.) qui nous intéresse:

$$
\begin{aligned}
{\left[\frac{d \sigma}{d \Omega}(q, E)\right]_{\mathrm{X}}^{\text {total }} } & =\left[\frac{d \sigma}{d \Omega}(q, E)\right]_{\mathrm{X}}^{\mathrm{R} . \mathrm{T} .}+\left[\frac{d \sigma}{d \Omega}(q, E)\right]_{\mathrm{X}}^{\text {Fluo }}+\left[\frac{d \sigma}{d \Omega}(q, E)\right]_{\mathrm{X}}^{\text {Raman }} \\
& +\left[\frac{d \sigma}{d \Omega}(q, E)\right]_{\mathrm{X}}^{\text {Compton }}+\left[\frac{d \sigma}{d \Omega}(q, E)\right]_{\mathrm{X}}^{\text {autres }}
\end{aligned}
$$

où l'on montre explicitement la dépendance non seulement en $q$ mais aussi en énergie incidente $E$, et les "autres" processus ne sont pertinents qu'aux enérgies très elevées du rayon $\mathrm{X}$. La section efficace Rayleigh-Thomson se décompose par la suite de la manière déjà présentée dans l'Eq. (29):

$$
\begin{aligned}
\frac{1}{N}\left[\frac{d \sigma}{d \Omega}(q, E)\right]_{\mathrm{X}}^{\mathrm{R} . \mathrm{T} .} & =S_{\mathrm{T}}(q, E)+\left(\overline{\left(\overline{b(q, E)^{2}}\right.}-\overline{b(q, E)}^{2}\right) \\
& =F_{\mathrm{T}}(q, E)+\overline{b(q, E)^{2}}
\end{aligned}
$$

où la dépendance en $(q, E)$ des longueurs de diffusion $b$ (voir la prochaine sous-section) est naturellement apporté à $S_{\mathrm{T}}$ et $F_{\mathrm{T}}$ par les équations (27) et (21).

Lorsque l'énergie incidente $E$ du rayon X devient suffisante pour sortir un électron par l'effet photoélectrique, on parle d'un seuil d'absorption ( $\left.E \geq E_{\text {seuil }}\right)$ pour la couche électronique où résidait l'électron (le plus souvent la couche $\mathrm{K}$ pour des mesures de diffraction). La fluorescence est une désexcitation atomique après cette absorption et émet des rayons X d'énergie $E_{\text {fluo }}<E_{\text {seuil }}$ de manière isotrope.

La diffusion Raman résonante a lieu jusqu'en dessous d'un seuil d'absorption où $E$ n'est pas suffisante pour sortir un électron mais le rayon $\mathrm{X}$ peut interagir avec un état virtuel de cet électron et ainsi perdre de l'énergie. Cette perte $E-E_{\mathrm{R}}$ reste constante lorsque $E$ approche $E_{\text {seuil }}$ mais l'interaction devient de plus en plus probabable d'où l'intensité Raman augmente beaucoup très près du seuil. Le seuil atteint, $E_{\mathrm{R}}$ devient $E_{\text {fluo }}$ et l'on peut effectivement penser à la diffusion Raman résonante, qui est isotrope, comme une "presque fluorescence". 
La diffusion Compton résulte d'une collision "balles de billiards" entre le rayon X et un électron quasiment libre (e.g. un électron de valence d'un métal). L'intensité Compton est fonction de la perte d'énergie du rayon $\mathrm{X}$ qui est à son tour fonction de l'angle de diffusion $2 \theta$ :

$$
\Delta \lambda=\left(\lambda_{f}-\lambda_{i}\right)=\frac{h}{m c}[1-\cos (2 \theta)],
$$

où l'énergie incidente $E=h c / \lambda_{i}$ et l'on ignore ici les effets d'énergie de liaison. La section efficace de la diffusion Compton, intégrée sur toutes les échanges d'énergie, est

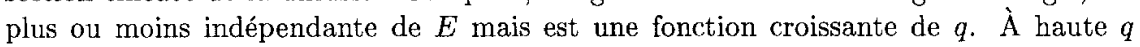
elle tend vers une valeur d'environ $1 / Z$ de l'intensité de la diffusion "self" de l'atome: $b(q \sim 0, E)^{2}$. Pourtant, c'est plutôt à petit $q$ où il peut être très difficile d'éliminer la diffusion Compton car sa spectre en énergie s'approche de très près de l'énergie incidente $E[25]$.

Bien qu'une discrimination électronique en énergie (auprès du détecteur) suffit souvent pour éliminer la fluorescence et parfois pour enlever une bonne partie de la diffusion Compton, l'énergie de la diffusion Raman est trop près de l'énergie incidente/élastique $E$ et requiert la presence d'un analyseur monocristallin devant le détecteur pour être bloquée (i.e. par non satisfaction de la condition Bragg). Par exemple, $E-E_{\mathrm{R}}$ n'est que $56 \mathrm{eV}$ pour le seuil $\mathrm{K}$ de Fe à $7112 \mathrm{eV}$, et en général $\left(E-E_{\mathrm{R}}\right) / E \approx 1 \%$ pour les métaux de transition.

En sommaire, pour bien faire la diffraction des rayons $\mathrm{X}$ il faut bloquer ou soustraire les contributions à $d \sigma / d \Omega$ non-voulues afin d'isoler la partie Rayleigh-Thomson et puis la normaliser (e.g. par l'Eq. (41) en connaissant les $b(q, E)$ ). Notons qu'il n'y a pas d'équivalent d'une normalisation par vanadium pour les rayons $\mathrm{X}$.

\subsection{Détermination des facteurs de structure partiels par AXD}

De manière analogue à celle de la substitution isotopique, la technique de diffraction anomale des rayons X $\left(\mathrm{AXD}\right.$ ) consiste à mesurer $S_{\mathrm{T}}(q)$ (ou bien $F_{\mathrm{T}}(q)$ ) sur des échantillons de structure identique mais ayant des distributions de longueurs de diffusion différentes. Les longueurs de diffusion pour les rayons $\mathrm{X}$ (symbol $f$ et non $b$ par convention) ne dépendent pas de l'isotope d'un élément $Z$ donné, mais de l'énérgie incidente du rayon $\mathrm{X}$ par l'effet anomale ou résonant:

$$
f(q, E)=r_{0}\left[Z M(q)+f^{\prime}(E)+i f^{\prime \prime}(E)\right]
$$

où $E$ est l'énergie incidente du rayon $\mathrm{X}, r_{0}=2.818 \mathrm{fm}$ le rayon classique d'un électron, $Z$ le nombre atomique, $M(q)$ la modulation du facteur de forme atomique qui varie de $1(q=0)$ à $0(q=\infty)$, et finalement $f^{\prime}=\Re\left(f_{\mathrm{a}}\right)$ et $f^{\prime \prime}=\Im\left(f_{\mathrm{a}}\right)$ sont les parties réelle et imaginaire du terme anomale $f_{\mathrm{a}}(E)=f^{\prime}(E)+i f^{\prime \prime}(E)$ (exprimés en unités de nombre d'électrons). On ignorera ici la légère dépendance en $q$ de $f_{\mathrm{a}}(E)$. Près d'un seuil d'absorption, $f^{\prime}$ diminue par quelques $e^{-}$ce qui permet de changer la longueur de diffusion (voir la Fig. 8). Par commodité le coté basse énergie $E \lesssim E_{\text {seuil }}$ est exploité pour minimiser $f^{\prime \prime}$ et ainsi l'absorption. Une tabulation des valeurs de $M(q), f^{\prime}(E)$ et $f^{\prime \prime}(E)$ pour les états neutre et ionique des éléments différents est donnée dans la 
$\mathrm{Fe}, \mathrm{Z}=26$
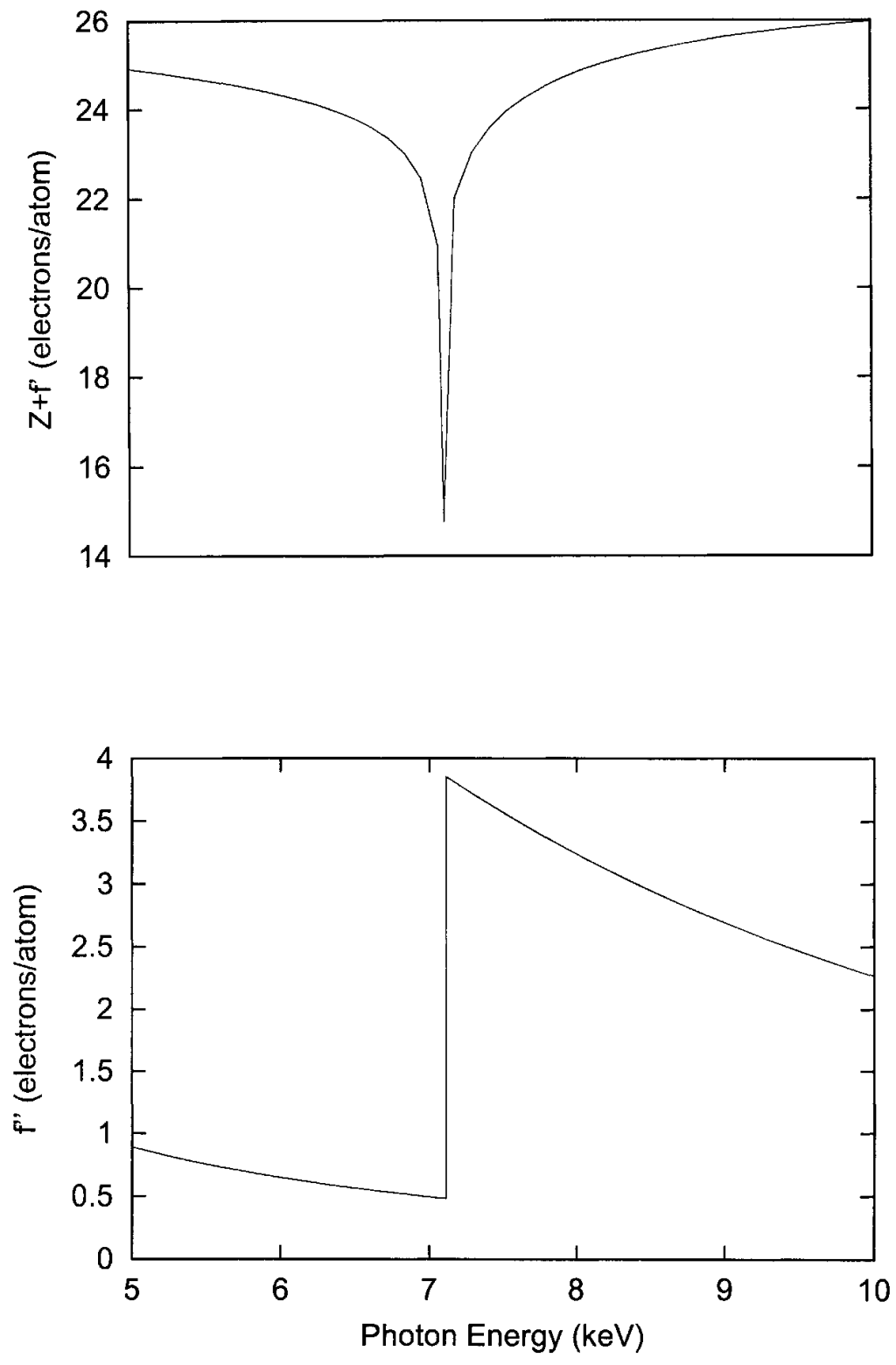

Figure 8. Variation de $f^{\prime}$ et de $f^{\prime \prime}$ près d'un seuil d'absorption des rayons $\mathrm{X}$, en unités de nombre d'électrons. 
Ref. [26]. Certaines informations et calculs pour $f(q, E)$ sont maintenant disponibles sur le web [27].

Pour l'AXD on n'a pas besoin d'isotopes donc un même échantillon suffit mais il faut varier l'énergie incidente $E$, e.g. à une source de rayonnement synchrotron. Nous pouvons ainsi reprendre les formules de la section précédente pour une série d'expériences de diffraction anomale des rayons $\mathrm{X}$ :

$$
F_{\mathrm{T} i}(q)=\sum_{\alpha, \beta}^{n} c_{\alpha} c_{\beta} f_{\alpha i}(q) f_{\beta i}^{*}(q) F_{\alpha \beta}(q)
$$

où $f_{\alpha i}(q)$ est la longueur de diffusion pour l'espèce atomique $\alpha$ à l'énergie incidente $E_{i}$. Cette équation montre explicitement la dépendence en $q$ des $f$, pour lesquels l'absorption importante des rayons $\mathrm{X}$ ne permet pas de négliger les parties imaginaires. Selon les éléments contenus dans l'échantillon, plusieurs seuils d'absorption peuvent être sondés. Notons que les $F_{\alpha \beta}(q)$ ici sont équivalents à ceux de l'Eq. (22) dépendant uniquement de la structure et non des longueurs de diffusion. Cette équivalence présuppose néanmoins une assez bonne sphéricité de la densité électronique $\rho_{\mathrm{e}^{-}}(r)$ des atomes.

Les $F_{\mathrm{T} i}(q)$ sont obtenus après normalisation et les corrections des $d \sigma / d \Omega$ discutées précédemment (bruit de fond, diffusion par l'environnement d'échantillon, attenuation, diffusion multiple, fluorescence, Raman, Compton). Pour un composé de 2 espèces atomiques la matrice pour l'AXD devient:

$$
\begin{aligned}
{\left[\begin{array}{l}
F_{\mathrm{T} 1}(q) \\
F_{\mathrm{T} 2}(q) \\
F_{\mathrm{T} 3}(q)
\end{array}\right] } & =\left[\begin{array}{lll}
c_{x}^{2} f_{x 1}^{2}(q) & c_{y}^{2} f_{y 1}^{2}(q) & c_{x} c_{y}\left[f_{x 1}(q) f_{y 1}^{*}(q)+f_{y 1}(q) f_{x 1}^{*}(q)\right] \\
c_{x}^{2} f_{x 2}^{2}(q) & c_{y}^{2} f_{y 2}^{2}(q) & c_{x} c_{y}\left[f_{x 2}(q) f_{y 2}^{*}(q)+f_{y 2}(q) f_{x 2}^{*}(q)\right] \\
c_{x}^{2} f_{x 3}^{2}(q) & c_{y}^{2} f_{y 3}^{2}(q) & c_{x} c_{y}\left[f_{x 3}(q) f_{y 3}^{*}(q)+f_{y 3}(q) f_{x 3}^{*}(q)\right]
\end{array}\right]\left[\begin{array}{l}
F_{x x}(q) \\
F_{y y}(q) \\
F_{x y}(q)
\end{array}\right] \\
& =\left[\begin{array}{lll}
a_{11}(q) & a_{12}(q) & a_{13}(q) \\
a_{21}(q) & a_{22}(q) & a_{23}(q) \\
a_{31}(q) & a_{32}(q) & a_{33}(q)
\end{array}\right]\left[\begin{array}{l}
F_{x x}(q) \\
F_{y y}(q) \\
F_{x y}(q)
\end{array}\right]
\end{aligned}
$$

où $f^{2}$ dénote $f f^{*}=|f|^{2}$. On voit facilement que chaque élément $a_{i j}$ est de valeur réelle (en barns). Le déterminant normalisé $|A(q)|_{n}$, maintenant en fonction de $q$, est toujours calculé après avoir divisé chaque rang $i$ de $\left[A_{i j}\right]$ par $\left[\sum_{j} a_{i j}^{2}(q)\right]^{1 / 2}$.

Les formules de première et deuxième différence deviennent alors pour l'AXD:

$$
\begin{aligned}
\Delta_{x(1-2)} F_{\mathrm{T}}(q) & \stackrel{\text { def }}{=} F_{\mathrm{T} 1}(q)-F_{\mathrm{T} 2}(q) \\
& =c_{x}^{2}\left[f_{x 1}^{2}(q)-f_{x 2}^{2}(q)\right] F_{x x}(q) \\
& +\sum_{\alpha \neq x}^{n} c_{\alpha} c_{x}\left[f_{\alpha}(q)\left(f_{x 1}^{*}(q)-f_{x 2}^{*}(q)\right)+f_{\alpha}^{*}(q)\left(f_{x 1}(q)-f_{x 2}(q)\right)\right] F_{\alpha x}(q)
\end{aligned}
$$

et

$$
\begin{aligned}
\Delta_{x}^{2} F_{\mathrm{T}}(q) & \stackrel{\text { def }}{=} \frac{1}{2}\left[\left(f_{x 2}^{*}(q)-f_{x 3}^{*}(q)\right)+\left(f_{x 2}(q)-f_{x 3}(q)\right)\right]\left[\Delta_{x(1-2)} F_{\mathrm{T}}(q)\right] \\
& -\frac{1}{2}\left[\left(f_{x 1}^{*}(q)-f_{x 2}^{*}(q)\right)+\left(f_{x 1}(q)-f_{x 2}(q)\right)\right]\left[\Delta_{x(2-3)} F_{\mathrm{T}}(q)\right]
\end{aligned}
$$




$$
\begin{aligned}
= & {\left[\Re\left(f_{x 2}(q)\right)-\Re\left(f_{x 3}(q)\right)\right]\left[F_{\mathrm{T} 1}(q)-F_{\mathrm{T} 2}(q)\right] } \\
- & {\left[\Re\left(f_{x 1}(q)\right)-\Re\left(f_{x 2}(q)\right)\right]\left[F_{\mathrm{T} 2}(q)-F_{\mathrm{T} 3}(q)\right] } \\
= & c_{x}^{2}\left[\left[\Re\left(f_{x 2}(q)\right)-\Re\left(f_{x 3}(q)\right)\right]\left(f_{x 1}^{2}(q)-f_{x 2}^{2}(q)\right)\right. \\
& \left.\quad-\left[\Re\left(f_{x 1}(q)\right)-\Re\left(f_{x 2}(q)\right)\right]\left(f_{x 2}^{2}(q)-f_{x 3}^{2}(q)\right)\right] F_{x x}(q)
\end{aligned}
$$

où les dernières expressions ont la commodité d'impliquer uniquement les normes et les parties réelles des longueurs de diffusion $f$. Une différence fondamentale des équations (46) et (47) pour l'AXD par rapport à celles pour le NDIS est qu'il faut supposer ici que les $f_{\alpha}(q)$ des atomes $\alpha$ ne changent pas trop près du seuil de l'atome $x$, tandis que pour le NDIS ce problème ne se présente pas.

Au lieu d'être limité par la disponibilité d'isotopes dans le cas du NDIS, nous sommes pour l'AXD contraint donc par l'existence de seuils d'absorption $E_{\text {seuil }}$ des éléments $Z$ dans l'échantillon qui soient à la fois accessibles et assez élévees pour que la gamme de $q=4 \pi E_{\text {seuil }} \sin \theta / h c$ explorée soit suffisamment grande. Pour obtenir une bonne transformation Fourier des $S_{\alpha \beta}(q)=F_{\alpha \beta}(q)+1$ en $g_{\alpha \beta}(r)$, une gamme en $q$ d'au moins $10 \AA^{-1}$ est généralement nécessaire, ce qui est fourni par $E_{\text {seuil }} \approx 10 \mathrm{keV}$. Les éléments ayant des énérgies d'absorption entre $10 \mathrm{keV}$ et $50 \mathrm{keV}$ (ce dernier étant souvent le maximum possible aux synchrotrons de 3ème génération) varient de $\mathrm{Ga}(Z=31)$ à $\mathrm{Gd}(Z=64)$ pour le seuil $\mathrm{K}$, et de $\operatorname{Tm}(Z=69)$ à $\mathrm{U}(Z=92)$ pour les seuils $\mathrm{L}$ (offrant une variation de $f^{\prime}$ plus faible que pour $\mathrm{K}$ ). On voit donc que l'AXD est une technique mieux exploitée pour les éléments de $Z>30$.

Pour les éléments de $Z$ important le contraste en longueur de diffusion offert par l'AXD est généralement comparable à celui pour le NDIS. La commodité d'un seul échantillon pour l'AXD est compensée par le fait que l'analyse des données est moins simple que dans le cas du NDIS, due aux processus Compton et Raman, aux effets d'attenuation et de diffusion multiple plus importants, et à la dépendence en $q$ de la matrice résultant du facteur de forme atomique $M(q)$ qui est d'ailleurs légèrement fonction de la structure chimique du composé. La forte décroissance de ce facteur $M(q)$ aux grandes valeurs de $q$, en raison des larges tailles des nuages d'électrons atomiques, mène naturellement à une résolution dans l'espace réel (i.e. pour $g(r))$ qui est inférieure à celle obtenable par diffraction des neutrons. Finalement, la variation de $f^{\prime}$ près d'un seuil d'absorption n'est généralement pas mesurable ni calculable avec une bonne précision et dépend un peu de l'environnement chimique de l'élément.

La Ref. [28] donne un bon exemple d'une expérience d'AXD portant des informations sur la structure d'hydration de l'anion $\mathrm{Br}^{-}$grâce à l'obtention d'une fonction de première différence $\Delta_{\mathrm{Br}} F_{\mathrm{T}}(q)$ et sa transformation Fourier.

\section{LA DIFFRACTION DES NEUTRONS ET DES RAYONS X COUPLÉE}

Puisque les longueurs de diffusion pour les neutrons (dépendantes du poids atomique et de l'interaction nucléaire) et celles pour les rayons $\mathrm{X}$ (dépendantes du nombre atomique 
et de l'interaction électrique) ne sont pas a priori corrélées (voir la Fig. 9), une combinaison ou couplage des mesures de diffraction par ces 2 techniques devrait offrir davantage de contraste entre les diffractogrammes $S_{\mathrm{T}}(q)$ pour la détermination des facteurs de structures partiels $S_{\alpha \beta}(q)$. La matrice liant les $S_{\mathrm{T}}(q)$ (ou $F_{\mathrm{T}}(q)$ ) avec les $S_{\alpha \beta}(q)$ (ou $\left.F_{\alpha \beta}(q)\right)$ comprendra donc des rangs "neutrons" (N) provenant de l'Eq. (33) et des rangs "rayons X" (X) provenant de l'Eq. (45), l'ensemble devenant alors dépendant de $q$.

L'utilisation conjointe de ces 2 techniques permet donc d'exploiter non seulement 2 types de longueurs de diffusion ( $b$ et $f$ ) pour chaque élément de l'échantillon, mais aussi 2 façons indépendantes de varier les longueurs de diffusion (NDIS et AXD). Cette souplesse constitue la complémentarité essentielle des neutrons et des rayons X pour la détermination des $S_{\alpha \beta}(q)$ et donc des $g_{\alpha \beta}(r)$.

Cependant, la combinaison de ces 2 techniques engendre aussi quelques difficultés d'analyses des données. En particulier, on ne peut plus annuler certaines erreurs systématiques résiduelles par "soustraction" (i.e. par combinaisons linéaires des diffractogrammes dans la matrice), comme il est possible dans le cas des diffractogrammes obtenus par une seule technique. Il faut plutôt procéder à des corrections des sections efficaces $[d \sigma / d \Omega]_{\mathrm{X}}$ et $[d \sigma / d \Omega]_{\mathrm{N}}$ assez exactes pour que les erreurs systématiques résiduelles soient plus petites que la différence entre ces sections efficaces, c'est à dire, plus petites que le contraste. Par exemple, la soustraction de l'intensité provenant de l'environnement/container de l'échantillon ainsi que du bruit de fond exige un très bon traitment lorsque les diffractogrammes $\mathrm{X}$ et $\mathrm{N}$ sont combinés. De plus, la normalisation en absolu des $S(q)$, souvent difficile à faire, doit être assez précise. Il faut aussi confirmer que la même partie de l'échantillon est sondée par les 2 techniques, puisque les rayons $\mathrm{X}$ sont généralement plus sensibles à la surface de l'échantillon (encore en raison de plus d'absorption). Finalement, la différence en résolution [29] des 2 appareillages $X$ et $\mathrm{N}$ nécessite éventuellement de (dé)convoluer les diffractogrammes préalablement au calcul matriciel. À part ces facteurs expérimentaux, il faut également se rendre compte qu'une éventuelle non-sphéricité importante de la densité électronique $\rho_{\mathrm{e}^{-}}(r)$ des atomes remettrait en question l'isotropie du facteur de forme $M(q)$ - c'est à dire, les neutrons et les rayons $\mathrm{X}$ ne diffracteraient plus de la même structure.

Pour illustrer l'avantage de la combinaison des diffractogrammes $\mathrm{N}$ et $\mathrm{X}$, on prend l'exemple des facteurs de structure partiels de l'eau: $S_{\alpha \beta}(q)$ ou $\alpha, \beta=\mathrm{H}, \mathrm{O}$. D'un intérêt particulier est le facteur de structure oxygène-oxygène $S_{\mathrm{OO}}(q)$ portant des informations sur la structure (intermoléculaire) des liaisons d'hydrogène (i.e. H-bonds). La diffraction des rayons X "voit" principalement les atomes d'oxygène $(Z=8)$ au dépit des atomes d'hydrogène $(Z=1)$, mais le seuil d'absorption de l'oxygène est trop faible en énergie pour donner une gamme suffisante en $q$ (de même pour le seuil d'hydrogène) donc l'AXD n'est pas rentable. Le NDIS, quant à lui, n'est pas très bien adapté à la détermination de $S_{\mathrm{OO}}(q)$ car il n'existe pas d'isotopes d'oxygène appropriés. Par contre, la combinaison du NDIS sur l'hydrogène (i.e. 2 échantillons ayant des deutérations différentes) plus un diffractogramme des rayons X "normale" (i.e. sans faire jouer l'effet anomale) devrait augmenter beaucoup le contraste et ainsi permettre une robuste détermination de tous les 3 facteurs de structure partiels.

D'abord on considère le contraste lorsque seulement le NDIS est employé. Pour minimiser la diffusion incohérente et multiple provenant de l'hydrogène, on se limite à une deutération mimimum de $60 \%$. Les 3 échantillons de NDIS sont alors de compositions: 1) $0 \% \mathrm{H}, 2) 20 \% \mathrm{H}$, et 3) $40 \% \mathrm{H}$ sur les sites $Z=1$. Notons que les 


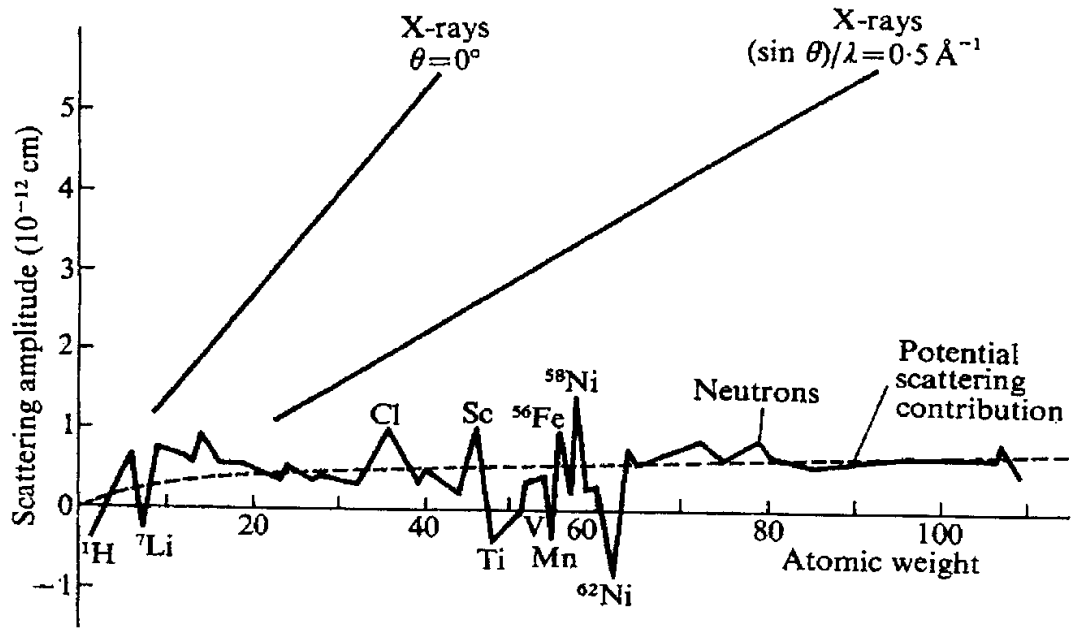

Figure 9. Les grandeurs relatives des longueurs de diffusion $b$ (neutrons) et $f$ (rayons $\mathrm{X}$ ), ici dénotées "scattering amplitude", en fonction du poids atomique. Notons la dépendance en $q \propto \sin \theta / \lambda$ pour les rayons $\mathrm{X}$ mais non pour les neutrons. D'après Bacon (1975).

deutérons $\mathrm{D}={ }^{2} \mathrm{H}$ se substituent pour les ${ }^{1} \mathrm{H}$ de manière aléatoire, sans corrélations en positions, pour assurer la bonne longueur de diffusion moyenne $\bar{b}$ des sites $Z=1$. Après que les corrections discutées précédemment sont apportées aux 3 diffractogrammes $\mathrm{N}$ et la partie "self" est soustraite, les fonctions d'interférence totaux $F_{T i}(q)$ sont obtenues, ayant des unités en barns et convergant à 0 pour $q \rightarrow \infty$. La matrice qui les lie aux facteurs de structure partiels (sans dimension) $S_{\alpha \beta}(q)-1=F_{\alpha \beta}(q)$ est alors:

$$
\left[\begin{array}{c}
F_{\mathrm{T}, 0.0 \mathrm{H}}(q) \\
F_{\mathrm{T}, 0.2 \mathrm{H}}(q) \\
F_{\mathrm{T}, 0.4 \mathrm{H}}(q)
\end{array}\right]=\left[\begin{array}{ccc}
19.78 & 3.742 & 17.21 \\
9.356 & 3.742 & 11.83 \\
2.7919 & 3.742 & 6.4642
\end{array}\right]\left[\begin{array}{l}
S_{\mathrm{HH}}(q)-1 \\
S_{\mathrm{OO}}(q)-1 \\
S_{\mathrm{OH}}(q)-1
\end{array}\right],
$$

dont le déterminant normalisé $|A|_{\text {NDIS }}=0.0236$ est typique pour le NDIS, mais l'absence de substitution isotopique sur l'oxygène rend le calcul assez peu sensible à $S_{\mathrm{OO}}(q)$.

Maintenant on remplace le diffractogramme $\mathrm{N}$ de l'échantillon de $40 \% \mathrm{H}$ par une diffractogramme X à une énergie incidente de $E=20 \mathrm{keV}$. La matrice devient alors dépendante en $q$ et à $q=5 \AA^{-1}$ elle est donnée par:

$$
\left[\begin{array}{c}
F_{\mathrm{T}, 0.0 \mathrm{H}}(q) \\
F_{\mathrm{T}, 0.2 \mathrm{H}}(q) \\
F_{\mathrm{T}, \mathrm{X}}(q)
\end{array}\right]=\left[\begin{array}{ccc}
19.78 & 3.742 & 17.21 \\
9.356 & 3.742 & 11.83 \\
0.0596 & 7.973 & 1.379
\end{array}\right]\left[\begin{array}{c}
S_{\mathrm{HH}}(q)-1 \\
S_{\mathrm{OO}}(q)-1 \\
S_{\mathrm{OH}}(q)-1
\end{array}\right],
$$


qui a un déterminant normalisé de $|A|_{\text {NDIS }+\mathrm{X}}=0.159$, soit presque 7 fois $|A|_{\text {NDIS }}$, et indiquant un conditionnement exceptionnel pour l'inversion de la matrice:

$$
\left[\begin{array}{c}
S_{\mathrm{HH}}(q)-1 \\
S_{\mathrm{OO}}(q)-1 \\
S_{\mathrm{OH}}(q)-1
\end{array}\right]=\left[\begin{array}{ccc}
0.1683 & -0.2491 & 0.0379 \\
0.0230 & -0.0495 & 0.1379 \\
-0.1404 & 0.2971 & -0.0736
\end{array}\right]\left[\begin{array}{c}
F_{0.0 \mathrm{H}}(q) \\
F_{0.2 \mathrm{H}}(q) \\
F_{\mathrm{X}}(q)
\end{array}\right]
$$

qui détermine les $S_{\alpha \beta}(q)$ et puis les $g_{\alpha \beta}(r)$ par transformation Fourier. Le contraste entre des $F_{\mathrm{T}}(q)$ dans la matrice NDIS $+\mathrm{X}$ est en effet tellement bon qu'il devrait pouvoir dépasser largement les éventuelles erreurs systématiques résiduelles de ceux-ci.

Bien que l'eau soit un exemple impressionnant en ce qui regarde l'amélioration du contraste lorsque la diffraction des rayons $\mathrm{X}$ est apportée à la détermination de ses facteurs de structure partiels, ce système de faible nombre atomique $Z$ présente pourtant plusieurs problèmes. Les distributions électroniques des atomes $\mathrm{H} / \mathrm{D}$ et $\mathrm{O}$ ne sont guère sphériques dans la molécule d'eau, et par conséquent les neutrons et les rayons $\mathrm{X}$ ne voient pas exactement les mêmes centres de diffusion. Coté neutrons, les faibles masses des atomes $\mathrm{H}$ et $\mathrm{D}$ engendrent d'importantes et imprécises corréctions de diffusion inélastique, tandis que la forte diffusion incohérente des atomes $\mathrm{H}$ produit beaucoup de diffusion multiple. De plus, le facteur de 2 entre les masses des atomes $\mathrm{H}$ et $\mathrm{D}$ mène à de subtiles différences structurales entre l'eau protonée et deutériée à une température donnée [30]. Coté rayons $\mathrm{X}$, le très faible nombre atomique des atomes $\mathrm{H}$ donne un rapport très défavorable (proportionnel à $Z$ ) des intensités de diffusion Rayleigh-Thomson et de diffusion Compton - et la contamination par cette dernière est encore plus difficile à calculer précisément car très dépendante de la nature de la liaison complexe de l'électron provenant de l'atome de l'hydrogène. Bref, le bon couplage des techniques de NDIS et de diffraction des rayons $\mathrm{X}$ s'avère difficile pour des système de faible nombre atomique.

Un meilleur exemple de l'aide apporté par les rayons $\mathrm{X}$ dans la détermination des facteurs de structure partiels est l'étude par Barnes, et al, sur le composé binaire liquide TlSe [8]. Les distributions électroniques des atomes $\mathrm{Tl}$ et Se étant assez sphériques dans ce système de nombres atomiques $Z$ moyens, et les effets de diffusion inélastique (neutrons) et de diffusion Compton (rayons $\mathrm{X}$ ) étant assez faibles, la combinaison des techniques de NDIS et de diffraction des rayons $\mathrm{X}$ peut ici être effectuée de manière assez propre. La Fig. 10 montre les résultats pour les trois $S_{\alpha \beta}(q)$ lorsque seulement le NDIS est employé. On voit que la précision statistique de l'expérience, menée sur le diffractomètre D4b à l'ILL, n'est pas vraiment suffisante pour une adéquate résolution des facteurs de structure partiels. Effectivement, le déterminant normalisé $|A|_{\text {NDIS }}=0.005$ met l'expérience à la limite de faisabilité. La Fig. 11 montre les résultats lorsque l'un des diffractogrammes $\mathrm{N}$ a été remplacé par un diffractogramme $\mathrm{X}$. Le déterminant normalisé devient alors $|A|_{\text {NDIS }+\mathrm{X}}=0.088$, soit plus qu'un facteur de 17 d'amélioration. Ces facteurs de structure partiels améliorés ont donné les $g_{\alpha \beta}(r)$ de Fig. 12 après transformation Fourier.

Une autre étude encore plus récente a été menée sur le composé $\mathrm{Ga}_{2} \mathrm{Te}_{3}$ liquide pour laquelle et le NDIS et l'AXD ont été exploités [9]. La substitution isotopique sur $\mathrm{Te}$ a fourni 3 diffractogrammes $\mathrm{N}$, et l'effet anomale près du seuil $\mathrm{K}$ de $\mathrm{Te}$ a fourni 


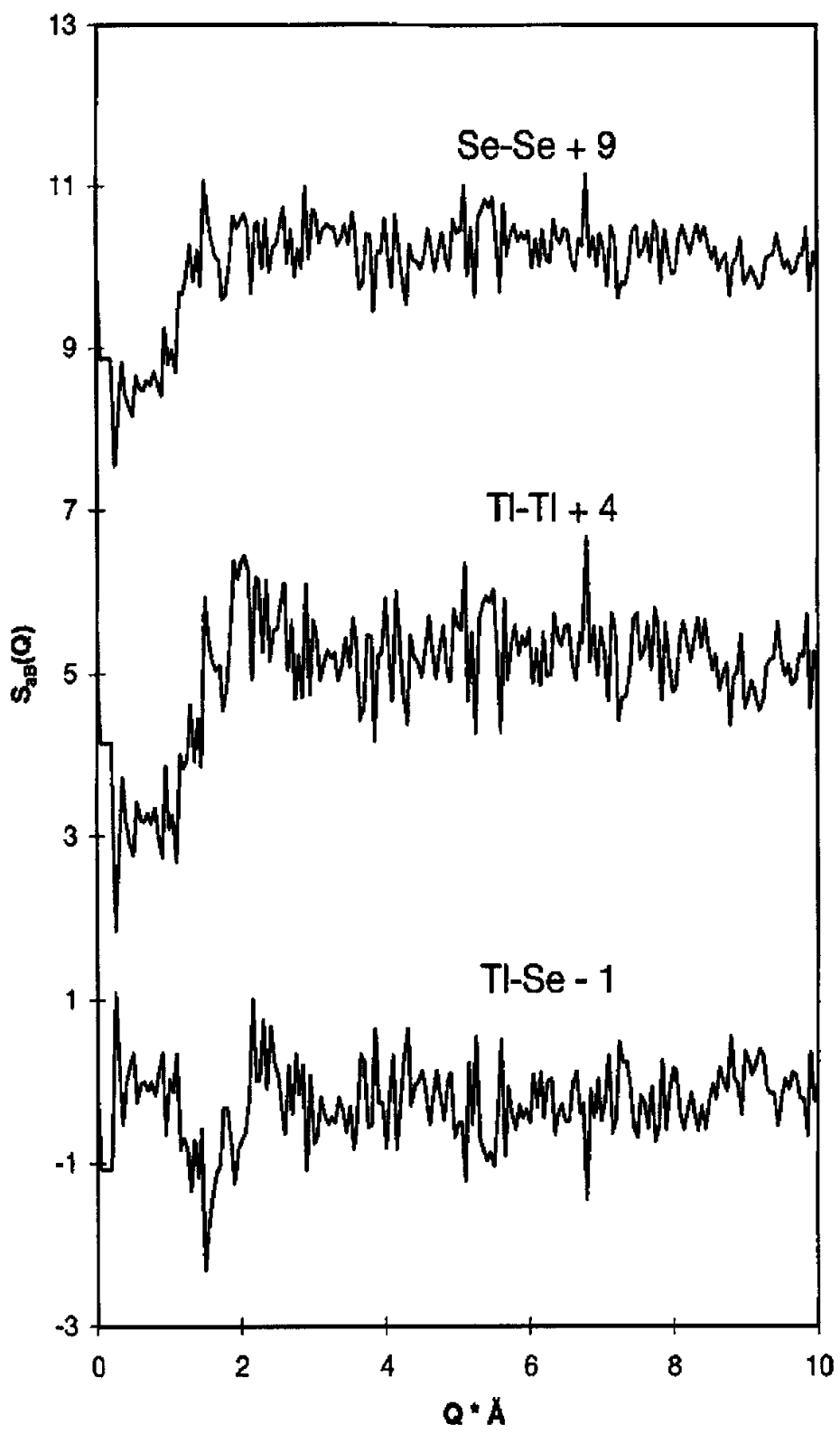

Figure 10. Facteurs de structure partiels $S_{\alpha \beta}(q)$ du composé binaire liquide TlSe obtenus par NDIS seulement. 


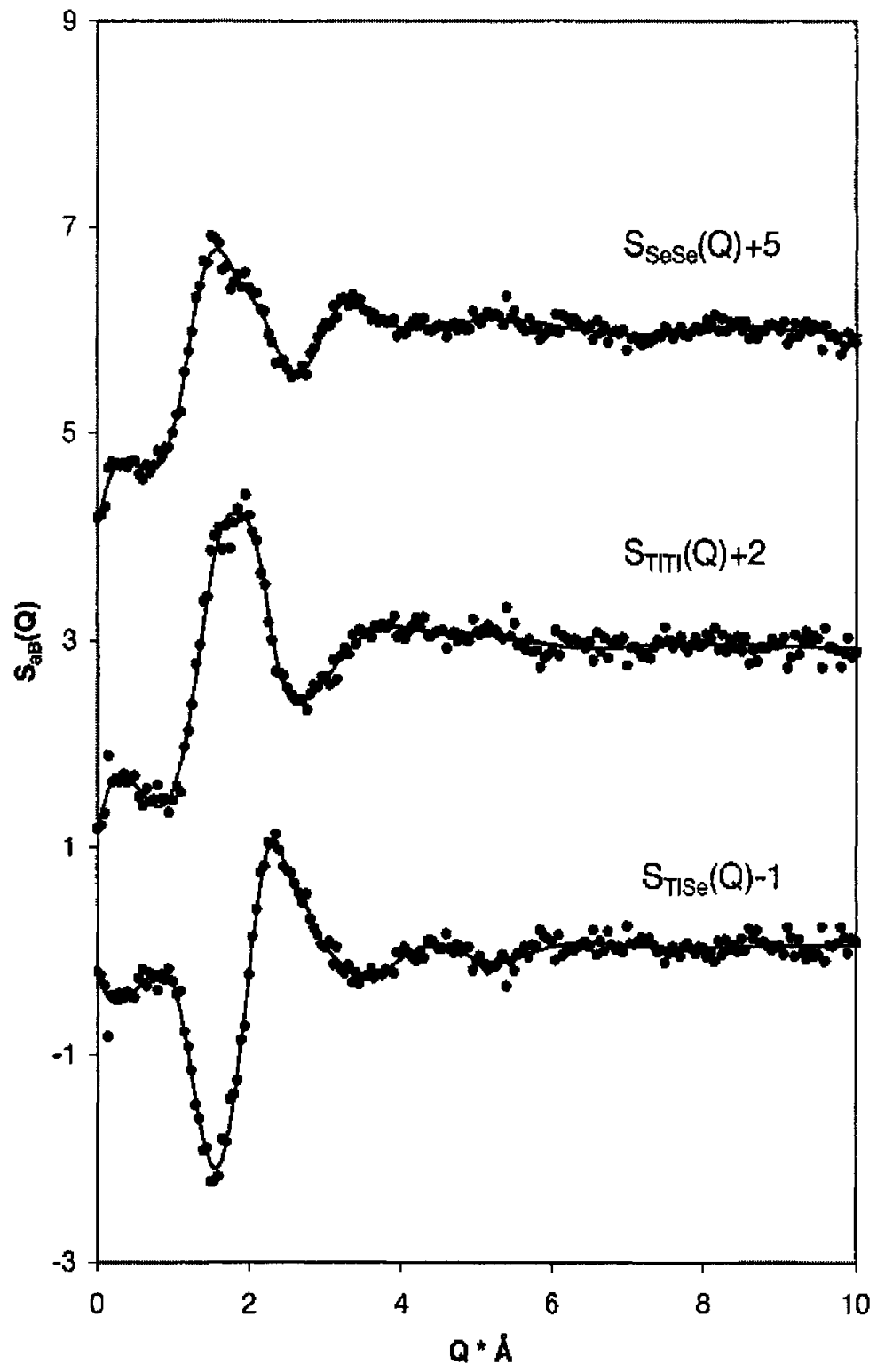

Figure 11. Facteurs de structure partiels $S_{\alpha \beta}(q)$ du composé binaire liquide TlSe obtenus par NDIS plus diffraction X. 


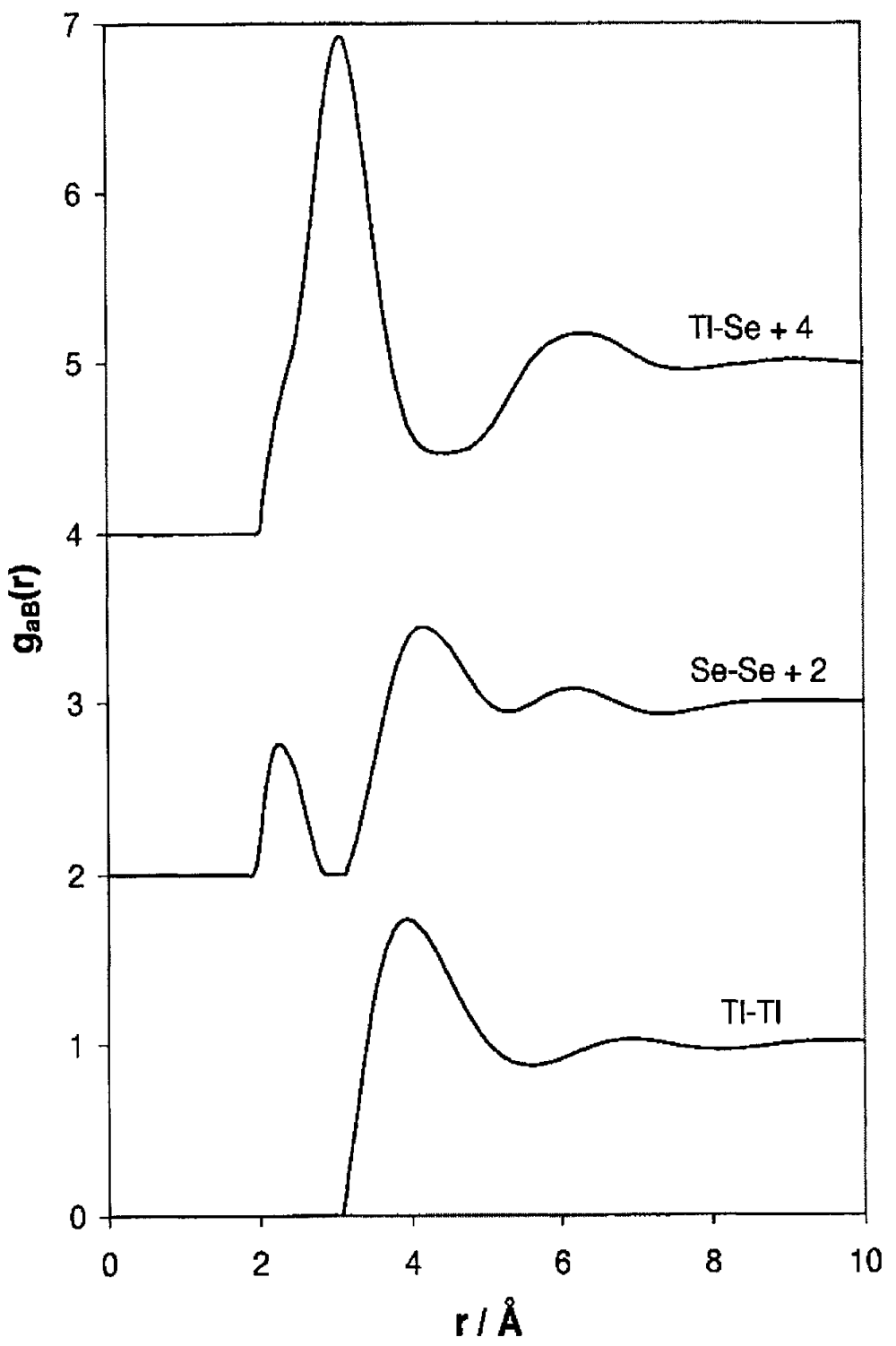

Figure 12. Fonctions de distribution de paires partielles $g_{\alpha \beta}(r)$ du composé binaire liquide TISe obtenus par NDIS plus diffraction X. Les 2 courbes du haut ont été décalées en ordonnée de 2 et de 4 . 
2 diffractogrammes X. Les 3 facteurs de structure partiels ont été calculés de ces 5 diffractogrammes par une méthode de moindres carrés, le SVD $=$ (overdetermined) Singular Value Decomposition [31].

Finalement, de bons résultats pour des fonctions de première différence et pour des facteurs des structure partiels ont été obtenus pour les verres covalents PSe [10] et $\mathrm{GeO}_{2}[11]$ en exploitant les diffractions $\mathrm{N}$ et $\mathrm{X}$.

En conclusion, on a vu que la combinaison des techniques de diffraction des neutrons et de diffraction des rayons $\mathrm{X}$ peut apporter de forts avantages à la détermination de facteurs de structure partiels, e.g. l'accessibilité à un plus grand nombre d'éléments ainsi qu'un meilleur contraste en longueur de diffusion, par rapport à l'exploitation de soit NDIS soit AXD seul. Par contre, il reste du travail à faire en ce qui concerne la mise au point des techniques de mesure et d'analyse de données pour la diffraction des neutrons et des rayons $\mathrm{X}$ couplée, surtout pour des système ayant des atomes de faible nombre atomique.

\section{Références}

1. P. Chieux, J. Mol. Struct. 296, 177 (1993).

2. J.E. Enderby et A.C. Barnes, Rep. Prog. Phys. 53, 85 (1990).

3. P. Chieux, dans Neutron Diffraction, édité par H. Dachs (Springer-Verlag, Berlin, 1978), Topics in Current Physics 6, pp. 271-302.

4. A.C. Barnes, S.B. Lague, P.S. Salmon et H.E. Fischer, J. Phys. Condens. Matter 9 , 6159 (1997).

5. Ingrid Petri, Philip S. Salmon et Henry E. Fischer, Phys. Rev. Lett. 84, 2413 (2000); I. Petri, P.S. Salmon et H.E. Fischer, ILL Annual Report 1998, p. 52.

6. Yoshio Waseda, Novel application of anomalous (resonance) $x$-ray scattering for structural characterization of disordered materials (Springer-Verlag, Berlin, 1984), Lecture Notes in Physics 204.

7. J.B. Suck, D. Raoux, P. Chieux et C. Riekel (eds.), Methods in the determination of partial structure factors (World Scientific, Singapore, 1993).

8. A.C. Barnes, S.B. Lague, M.A. Hamilton, H.E. Fischer, A.N. Fitch et E. Doryhee, J. Phys. Condens. Matter. 10, L645 (1998).

9. P. Buchanan, A.C. Barnes, K.R. Whittle, M.A. Hamilton, A.N. Fitch et H.E. Fischer, Molec. Phys. 99, 767 (2001).

10. A.C. Barnes, M.A. Hamilton, P. Buchanan et M.L. Saboungi, J. Non-Cryst. Solids 250-252, 393 (1999). 
11. D.L. Price, M.L. Saboungi et A.C. Barnes, Phys. Rev. Lett. 81, 3207 (1998).

12. Voir, par exemple, http://www.isis.rl.ac.uk/disordered pour plus d'informations sur la diffraction des neutrons provenant d'une source pulsée.

13. A.C. Wright, J. Non-Cryst. Solids 159, 264 (1993); D.I. Grimley, A.C. Wright et R.N. Sinclair, J. Non-Cryst. Solids 119, 49 (1990).

14. Varley F. Sears, Neutron News 3, 26 (1992); les mêmes informations sont aussi disponibles à http://www.ncnr.nist.gov/resources/n-lengths.

15. Voir http://www.ill.fr/YellowBook/D4 pour plus d'informations sur le diffractomètre D4.

16. P.A. Egelstaff, An introduction to the liquid state (Academic Press, London and New York, 1967).

17. T.E. Faber et J.M. Ziman, Phil. Mag. 11, 153 (1965).

18. A.B. Bhatia et D.E. Thornton, Phys. Rev. B 2, 3004 (1970).

19. H.H. Paalman et C.J. Pings, J. Appl. Phys. 33, 2635 (1962).

20. Alan K. Soper et P.A. Egelstaff, Nucl. Instr. Methods 178, 415 (1980).

21. T. Bausenwein, H. Bertagnolli, K. Tödheide et P. Chieux, Nucl. Instr. Methods B 61, 527 (1991).

22. G. Placzek, Phys. Rev. 86, 377 (1952).

23. J.L. Yarnell, M.J. Katz, R.G. Wenzel et S.H. Koenig, Phys. Rev. A 7, 2130 (1973).

24. G.E. Bacon, Neutron Diffraction, 3rd edition (Clarendon Press, Oxford, 1975).

25. J.M. Tonnerre, thèse doctorale de l'Université de Paris-Sud (1989).

26. A.J.C. Wilson, International Tables for X-ray Crystallography, vol. C (Kluwer, London, 1995).

27. Voir http://physics.nist.gov/PhysRefData/FFast/Text/cover.html et aussi http://www-cxro.lbl.gov/optical_constants/asf.html.

28. S. Ramos, A.C. Barnes, G.W. Neilson, D. Thiaudiere et S. Lequien, J. Phys. Condens. Matter 11, A203 (1999).

29. L.W. Finger, D.E. Cox et A.P. Jephcoat, J. Appl. Cryst. 27, 892 (1994); B. van Laar et W.B. Yelon, J. Appl. Cryst. 17, 47 (1984); G. Caglioti, A. Paoletti et F.P. Ricci, Nucl. Instrum. and Meth. 9, 195 (1960); G. Caglioti, A. Paoletti et F.P. Ricci, Nucl. Instrum. 3, 223 (1958).

30. Y.S. Badyal, M.L. Saboungi, D.L. Price, S.D. Shastri, D.R. Haeffner et A.K. Soper, J. Chem. Phys. 112, 9206 (2000).

31. William H. Press, Saul A. Teukolsky, William T. Vetterling et Brian P. Flannery, Numerical Recipes in $C$, 2nd edition (Cambridge University Press, 1995). 